\title{
Notch signaling during development requires the function of awd, the Drosophila homolog of human metastasis suppressor gene $\mathrm{Nm} 23$
}

\author{
Marilena Ignesti ${ }^{1}$, Marilena Barraco ${ }^{1,5}$, Gouthami Nallamothu' ${ }^{2}$, Julie A Woolworth ${ }^{3}$, Serena Duchi ${ }^{1,6}$,
} Giuseppe Gargiulo ${ }^{1}$, Valeria Cavaliere ${ }^{1 *}$ and Tien $\mathrm{Hsu}^{2,4^{*}}$

\begin{abstract}
Background: The Drosophila abnormal wing discs (awd) belongs to a highly conserved family of genes implicated in metastasis suppression, metabolic homeostasis and epithelial morphogenesis. The cellular function of the mammalian members of this family, the Nm23 proteins, has not yet been clearly defined. Previous awd genetic analyses unraveled its endocytic role that is required for proper internalization of receptors controlling different signaling pathways. In this study, we analyzed the role of Awd in controlling Notch signaling during development.

Results: To study the awd gene function we used genetic mosaic approaches to obtain cells homozygous for a loss of function allele. In awd mutant follicle cells and wing disc cells, Notch accumulates in enlarged early endosomes, resulting in defective Notch signaling. Our results demonstrate that awd function is required before $\gamma$-secretase mediated cleavage since over-expression of the constitutively active form of the Notch receptor in awd mutant follicle cells allows rescue of the signaling. By using markers of different endosomal compartments we show that Notch receptor accumulates in early endosomes in awd mutant follicle cells. A trafficking assay in living wing discs also shows that Notch accumulates in early endosomes. Importantly, constitutively active Rab5 cannot rescue the awd phenotype, suggesting that awd is required for Rab5 function in early endosome maturation.

Conclusions: In this report we demonstrate that awd is essential for Notch signaling via its endocytic role. In addition, we identify the endocytic step at which Awd function is required for Notch signaling and we obtain evidence indicating that Awd is necessary for Rab5 function. These findings provide new insights into the developmental and pathophysiological function of this important gene family.
\end{abstract}

Keywords: Awd, Notch signaling, Endocytosis

\section{Background}

The Drosophila awd (abnormal wing discs) gene was identified in a genetic screen for genes involved in imaginal disc development [1,2]. It encodes the Drosophila homolog of human metastasis suppressor gene Nm23 [3,4]. The $N m 23$ gene family (also termed NME) consists of ten related genes in mammals [5] with the NME1 and NME2 isoforms most implicated in tumor progression and sharing about $78 \%$ of amino acid identity with the Awd protein.

\footnotetext{
*Correspondence: valeria.cavaliere@unibo.it; tienh@bu.edu

'Dipartimento di Farmacia e Biotecnologie, Alma Mater Studiorum Università

di Bologna, Via Selmi, 3, Bologna 40126, Italy

${ }^{2}$ Department of Medicine, Boston University School of Medicine, Boston,

Massachusetts 02118, USA

Full list of author information is available at the end of the article
}

During Drosophila development, awd is critical for epithelial morphogenesis [6] and has been linked to AMP kinaseregulated energy-sensing [7]. Human and murine $\mathrm{Nm} 23$ has been shown in cancer cell xenografts to inhibit metastasis, but not primary tumor growth [8]. On the other hand, in other cancer cohorts, particularly those of ovarian cancers, up-regulated $N m 23$ levels have been correlated with poor prognosis $[9,10]$, suggesting an oncogenic function. These discrepancies have so far been difficult to reconcile because the exact cellular function of $\mathrm{Nm} 23$ has remained unclear, although several molecular activities have been assigned to the Nm23 family of proteins. Nm23 belongs to a classic nucleoside diphosphate kinase (NDPK) family that generates nucleoside triphosphates using adenosine 
triphosphates (ATP) as a phosphate source [11], but other activities, such as histidine-dependent protein kinase [12-14], nuclease [15-18] and lipid bilayer-binding [19,20], have also been documented. Interestingly, in Drosophila, awd has been shown to interact genetically with dynamin to promote endocytosis [6,21], although it is not yet clear which endocytic process is regulated by awd. In neurons, awd has been shown to promote Dynamin-mediated neurotransmitter uptake at the neuromuscular junction [22]. Proper tracheal branching morphogenesis requires awd function to regulate internalization and signaling of the fibroblast growth factor receptor (FGFR) encoded by the breathless gene [23]. During oogenesis awd is downregulated in border cells to allow for accumulation of and chemotactic signaling from the platelet-derived growth factor/vascular endothelial growth factor (PDGF/VEGF) receptor (PVR) [24]. Awd also regulates Domeless signaling via modulating endocytosis [24]. Moreover, loss of awd function in the follicular epithelium causes mislocalization of $\beta$ catenin and DE-cadherin, resulting in over-accumulation of these adherens junction components and disruption of epithelial integrity [25]. During our analyses of awd function in the follicular epithelium, we also noted a proliferation abnormality in awd mutant cells that is reminiscent of the Notch signaling defect. This observation prompted us to revisit the original 'abnormal wing discs' phenotype, which led to the discovery of the classic 'notched wing' phenotype in flies carrying mosaic awd mutant clones. Notch pathway is a highly conserved cell-cell communication pathway and functions to regulate many different cellular processes during embryonic development and in adulthood [26]. Canonical Notch signaling requires binding of membrane-bound Notch receptor to membrane-bound ligand Delta/Serrate/ Lag2 (DSL) on the juxtaposed cells. The interaction triggers proteolytic cleavage in the extracellular juxtamembrane region of Notch (S2 cleavage), separating the ligand-bound extracellular domain and the membrane-bound NEXT (Notch EXternal Truncation) [27]. NEXT is then subjected to intra-membrane proteolysis by $\gamma$-secretase (S3 cleavage). The proteolysis releases the intracellular domain of Notch (NICD), which translocates into the nucleus and regulates transcription of target genes by association with transcriptional cofactors of the CBF1-Su(H)-Lag1 (CSL) family $[26,28-30]$. More recently, it has been shown that in some cell types, Notch entry into the endocytic pathway is critical for proper Notch activation and signaling [31-34]. Since Notch signaling may function either as a tumor suppressor or as an oncogene, depending on the tissue context [35], the functional relationship between Nm23/awd and Notch may provide important insights into the seemingly contradictory roles of $\mathrm{Nm} 23$ in tumor progression. In addition, elucidating the Notch signaling defect in awd mutant cells should also shed light on the awd action in the endocytic pathway.
In the present study, we show that awd function is required for proper Notch signaling in follicle cells and imaginal disc cells. Genetic studies reveal that in awd mutants, Notch is blocked from entry into late endosomes and accumulates in abnormal, Avalanche (Avl)-positive vesicles, precluding signal activation.

\section{Results}

Notch signaling requires awd function in follicle cells and imaginal disc cells

The Drosophila egg chamber consists of a 16-germ cell syncytium enveloped by a monolayer of follicular epithelium [36]. The process of proliferation and differentiation of the follicle cells is complex and under stringent control [37-39]. One critical event is the cessation of mitosis in midoogenesis. The proliferation of follicle cells occurs before stage 7 (up to 30 hours after the egg chamber buds off from the germarium at stage 2; total egg chamber development time from stage 2 to stage 14 is approximately 70 hours). Notch signaling that regulates cell proliferation in the follicle cells is activated at stage 6 , which results in down-regulation of cut and cyclin B, among other Notch target genes, and cessation of mitosis [40-43]. From stage 7 to 10A (approximately 3 hours after stage 6) the follicle cell chromosomes continue to duplicate three times to generate polyploidity (endocycles). Disruption of Notch signaling causes extension of the proliferative program beyond stage 6 and follicle cells go through additional cell divisions without cell growth, resulting in increased cell number but reduced cell size.

We have previously shown that awd is involved in regulating epithelial integrity of the follicle cells via its endocytic activity [25]. During the course of examining follicular function of $a w d$, we also noticed that at later stages (after stage 8) the awd mutant clones often contain more numerous but smaller cells, suggesting faulty Notch signaling (Figure 1A). Since awd null alleles are lethal, the phenotypes in follicle cells, an adult tissue, are generated by mitotic recombination using the FLP/FRT system [44]. In this report, we employed different genetic methods that allow for induced mitotic recombination using temporal or tissue-specific expression of the recombinase FLP [45] or allow for co-expression of other transgenes in the awd mutant clones using the mosaic analysis with a repressible cell marker (MARCM) system [46]. While specific genetic strategies will be pointed out when appropriate, it is worth noting that the Notch phenotypes generated are consistent regardless of the FLP/FRT variations.

Immunostaining with the mitotic marker phosphorylated histone $\mathrm{H} 3$ (p-H3) shows that awd mutant cells continue to divide after stage 6 . In wild-type follicle cells $\mathrm{p}-\mathrm{H} 3$ positive cells are detectable only up to stage 6 of oogenesis (Figure 1B). Note that $\mathrm{p}-\mathrm{H} 3$ is only observed in $\mathrm{M}$ phase. Since mitosis of follicle cells is not synchronized, 


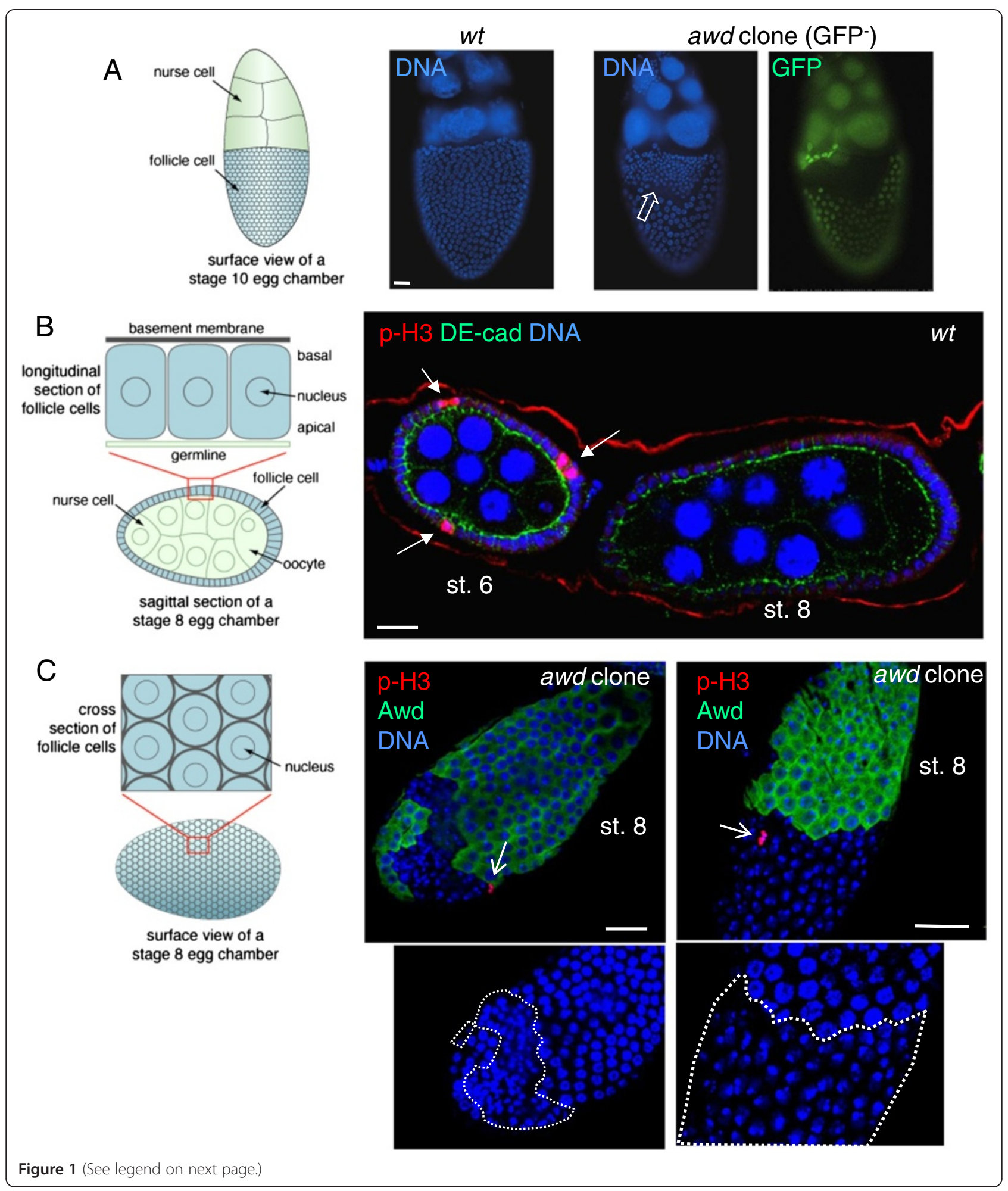


(See figure on previous page.)

Figure 1 Dysregulated proliferation in awd mutant follicle cells. (A-B) Control egg chambers were dissected from yw (representing wild-type) females. Egg chambers containing awd clones (no GFP) were dissected from hs-flp; +/t; Ubi-GFP, FRT ${ }^{82 B} / F R T^{82 B}$, awd ${ }^{2 A 4}$ females. (A) In follicular epithelium, awd mutant clones show more numerous but smaller nuclei (empty block arrow) in comparison with the adjacent normal follicle cells and with yw egg chambers (left). To visualize nuclei the stage 10 egg chambers were stained with DAPI (blue). (B) yw egg chambers were stained for p-H3 (red), DE-cadherin (green) and DNA (blue). p-H3 detects mitotic cells only in pre-stage 7 eggs (arrows). (C) Egg chambers containing awd clones were dissected from $y w$; en2.4-Gal4 ${ }^{22 C}$, UAS-flp/+; FRT $T^{82 B} / F R T^{82 B}$, awd $d^{2 A 4}$, and stained for p-H3 (red), Awd (green) and DNA (blue). In awd mutant clones (lack of Awd expression), p-H3 positive cells can be detected post-stage 6 (sharp arrows). Duplicate images showing only nuclear staining (insets) highlight the smaller nuclei in awd mutant clones (dashed lines). Schematic representations of the positioning and viewing of follicle cells in the egg chamber are shown in the left side of A, B and C. Bars are $20 \mu \mathrm{m}$. DAPI, 4',6-diamidino-2-phenylindole; p-H3, phosphorylated histone H3.

only a few cells are stained at any given time. In awd mutant cells $\mathrm{p}-\mathrm{H} 3$ staining is detectable after stage 6 (Figure 1C). Again, these awd mutant cells have smaller nuclei (insets in Figure 1C). Consistent with increased proliferation in awd mutant follicle cells, prolonged expression of the mitotic marker cyclin B was also detected in these mosaic ovaries (GFP-negative cells are mutants in Figure 2A). Note that while cyclin B is absent in $a w d^{+}$cells, in awd mutant cells, cyclin B is not uniformly expressed at high levels. This is likely because the cell cycle is not synchronized in all follicle cells. In addition, the known Notch down-regulation target cut [40] is over-expressed in Awdnegative cells (Figure 2B). Compromised Notch signaling also results in expression of immature cell-fate markers in follicle cells beyond stage 6 . In wild-type egg chambers Fasciclin III (FasIII) is expressed in all follicle cells up to stage 3 of oogenesis and then becomes restricted to the polar follicle cells (PC in Figure 2C) at the anterior and posterior poles of the follicular epithelium. Reduction of Notch activity arrests follicle cells in an undifferentiated state and up-regulates FasIII expression [42]. Follicle cell clones mutant for awd show strong expression of FasIII after stage 6, indicating that they are defective in terminal differentiation (awd mutant cells lacking GFP expression in Figure 2C). Down-regulation of cut in wild-type follicle cells is mediated by Hindsight (Hnt), an up-regulation target of Notch $[40,47]$. To examine loss of Notch target gene expression, we used the MARCM method of clonal analysis, which results in GFP-expression in mutant cells, so as to ensure that lack of gene expression is not the result of cell death (Figure 2D,E). In contrast to wild-type follicle cells, the MARCM clone of awd mutant cells (GFP-positive) does not express Hnt after stage 6 (Figure 2D). To further confirm that Notch signaling is attenuated in awd mutant follicle cells, the expression of $\mathrm{GbeSu}(H)_{m 8}$-lac $Z$ transcriptional reporter for Notch activity [48] was examined. In MARCM awd clones, $\beta$-galactosidase staining is absent or strongly reduced (GFP-positive cells in Figure 2E).

The Notch signaling defect in awd mutant cells suggested a potential mechanism for the original defining phenotype of awd - abnormal wing discs, because during development Notch specifies the dorsal-ventral margin of the wing discs (which becomes the wing peripheral margin in the adult) and the vein-intervein boundary, and is important for disc cell proliferation. Loss of Notch function causes wing margin defects and widening of wing veins [26]. As shown in Figure 3, 72\% (18/25) of adult mosaic flies show typical Notch phenotypes in wings with 'notched' wing margins and wing vein thickening (Figure 3A-C). In wild type wing discs, activation of the Notch pathway at the dorsal-ventral boundary (Figure 3D) leads to the expression of target gene products, such as the signaling molecule Wingless (Wg) [49]. Loss of awd function abolished the Wg staining in third instar wing disc clones at the dorsal-ventral boundary (GFP-negative cells in Figure 3E). To further verify the Notch signaling defect, we examined $\mathrm{GbeSu}(\mathrm{H})_{m 8}-\mathrm{lacZ}$ reporter expression using a different mosaic fly generated by the MARCM system. Similar to our results in follicular epithelium, $\beta$-galactosidase expression in awd mutant clones (GFP-positive cells) in the dorsal-ventral boundary is lost (Figure 3F).

\section{awd function is required for signaling after the S2 cleavage of Notch}

In the egg chamber, Notch functions in the follicle cells while the ligand Delta is expressed in the abutting germline cells [42]. Since the $a w d^{j 2 A 4}$ clones were induced specifically in follicle cells, the defective Notch signaling in mutant follicle cells is not likely to be the result of a defect in Delta expression or endocytosis in the abutting germline cells. Also importantly, in Delta mutant NICD antibody-detected Notch accumulates on the follicle cell surface, which is consistent with the notion that ligand binding precedes intracellular trafficking and proteolytic processing of Notch [42].

To define the step where Notch signaling is stalled in awd mutant follicle cells we over-expressed NICD or NEXT in awd mutant follicle cells by using the MARCM system. NICD is the cytoplasmic domain of Notch that functions as a cytoplasmic, $\gamma$-secretase-independent constitutively active Notch, while NEXT is the truncation generated after the S2 cleavage devoid of the ligand-binding domain, S2 cleavage site, and the negative-regulatory region (NRR) [50,51]. NEXT is a membrane-bound, $\gamma$-secretasedependent, constitutively active form of Notch that can function without ligand but still requires intracellular proteolytic processing and trafficking [52]. To assess rescue of 


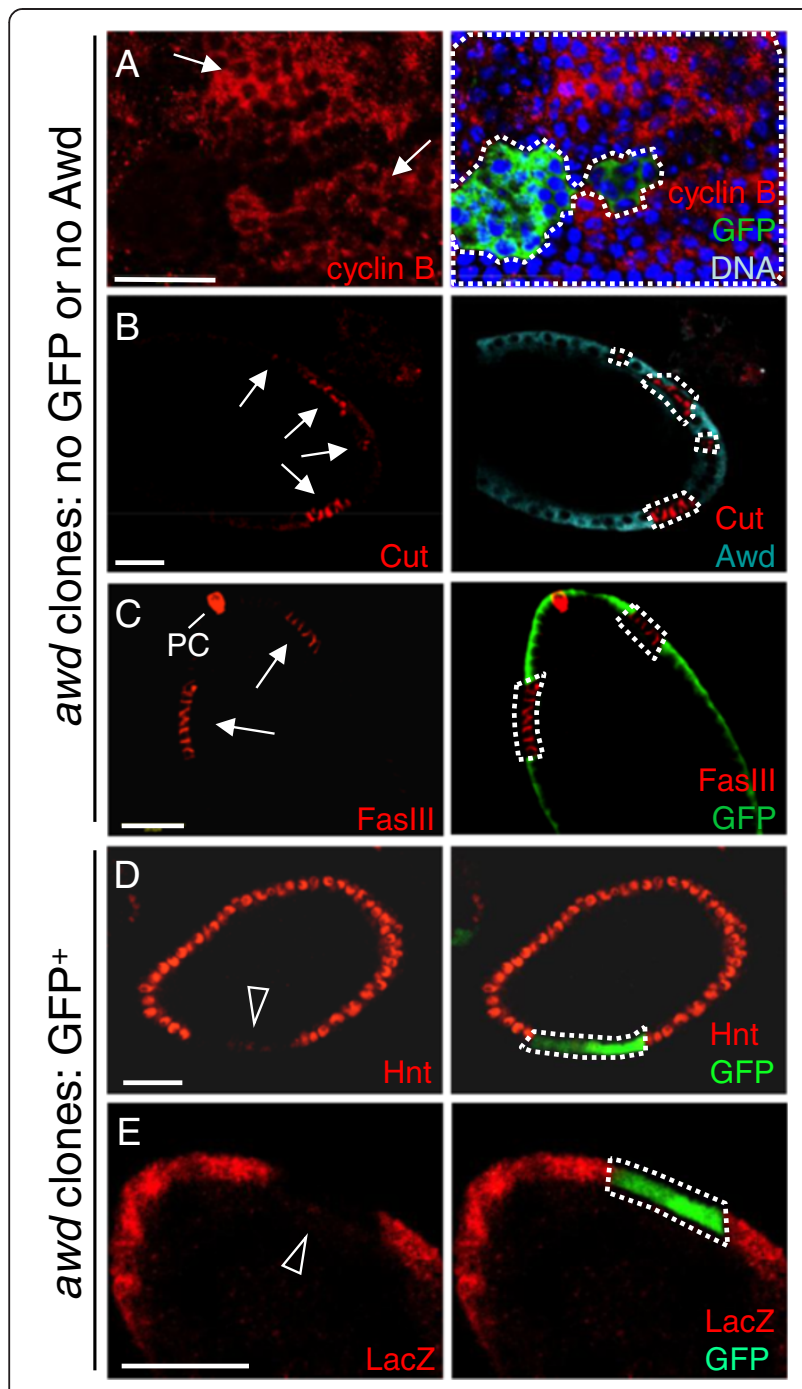

Figure 2 Altered expression of Notch signaling target genes in awd clones. (A and C) Stage 7-8 egg chambers were dissected from hs-flp; +/t; Ubi-GFP, FRT $T^{82 B} / F R T^{82 B}$, awd ${ }^{2 A 4}$ females, and the awd mutant clones were identified by lack of GFP staining (green).

(B, D-E) Stage 7 egg chambers were dissected from hs-flp/GbeSu(H) ms-lacZ; act-Gal4, UAS-GFP/+; FRT ${ }^{82 B}$, act-Gal80/FRT ${ }^{82 B}$, awd $d^{2 A 4}$

females. In (A-C) the awd mutant clones were identified by lack of Awd or GFP staining (green), while in (D-E) the awd mutant clones are GFP positive. In all panels, awd mutant clones are marked with dashed lines. (A) Cyclin B (red), (B) Cut (red) and (C) Fasciclin III (Faslll; in red) are normally negatively regulated by Notch signaling, but up-regulated in awd mutant clones (lack of GFP or Awd; arrows). (D) Hindsight (Hnt; in red) is normally induced by Notch signaling, and is down-regulated in awd clones (GFP-positive; empty arrowhead). (E) The lacZ reporter gene expression driven by Notch-activated Su(H) promoter [GbeSu(H) $)_{m 8}$ lacZ] is lost in awd clones (GFP-positive; empty arrowhead). Bars are $20 \mu \mathrm{m}$.

Notch signaling we analyzed the Hnt expression (no expression in awd mutant). In stage 7-8 awd clones overexpressing NICD (from the UAS-NICD transgene [53]) (GFP-positive cells in Figure 4A), 60.5\% of mutant cells express Hnt (199 out of 329 cells) (GFP-positive cells in
Figure 4B), representing a significant rescue of the lack of Hnt expression phenotype. This is also consistent with the observation that the over-expressed NICD is localized in the nuclei in a significant number of awd mutant follicle cells (Figure 4A). Furthermore follicle cells flp-out clones expressing the same NICD transgene also show enhanced Hnt expression at stage 7-8 [see Additional file 1: Figure S1A], as well as enhanced the size of nuclei at stage 10B (not shown) [54]. In contrast, expression of the UAS-NEXT transgene [55] in the awd clone (GFP-positive cells in Figure 4C) did not rescue Notch signaling as assessed by loss of $\mathrm{GbeSu}(\mathrm{H})_{m 8}$-lacZ expression (GFP-positive cells in Figure 4D) and loss of Hnt expression (GFP-positive cells in Figure 4E). The same transgene is able to upregulate the Hnt expression in flp-out clones of follicle cells [see Additional file 1: Figure S1B]. Note that the over-expressed NEXT accumulates in the intracellular vesicles (Figure 4C), consistent with the notion that internalization of surface Notch can occur in awd mutant cells but the subsequent vesicle trafficking is defective.

It has recently been shown that transmission of Notch signal requires proper intracellular trafficking, at least in Drosophila follicle cells and imaginal discs [32-34,55]. Therefore, our observed Notch processing and signaling defects may result from either defective proteolytic cleavage of Notch to release intracellular domain by $\gamma$ secretase or defective endocytic transport of Notch. We favor the latter mechanism since Awd has been shown to promote endocytosis of surface receptors in multiple tissues [21-25]. In addition, neither the expression level nor the punctate expression pattern of Presenilin [56-58], the catalytic component of the $\gamma$-secretase complex, are altered in awd mutant follicle cells [see Additional file 2: Figure S2]. To test the notion that the Notch signaling deficiency in awd mutant cells is the result of defective endocytosis, we next examined the localization of Notch receptor in awd follicle cell clones.

\section{Notch accumulates in endocytic vesicles in awd mutant cells}

While in $a w d^{+}$cells Notch is present in low abundance in small punctates, Notch accumulates in large vesiclelike aggregates near the apical surface in awd mutant clones in follicular epithelium (Figure 5A,B) and in wing discs (Figure 5C). Such Notch accumulation phenotype in awd mutant resembles that of mutants in avalanche (avl; which encodes Syntaxin) and rab5 [34,55,59], two gene functions required for maturation of early endosomes [59], but is different from the phenotype in dynamin mutant $\left(s h i^{t s}\right)$, in which Notch accumulates on the cell surface and in very large aggregates on apical and basal sides of the follicle cells (Figure 5D) as noted previously [60]. This pattern is likely because of the failure to deliver Notch to apical membrane via Dynamin- 


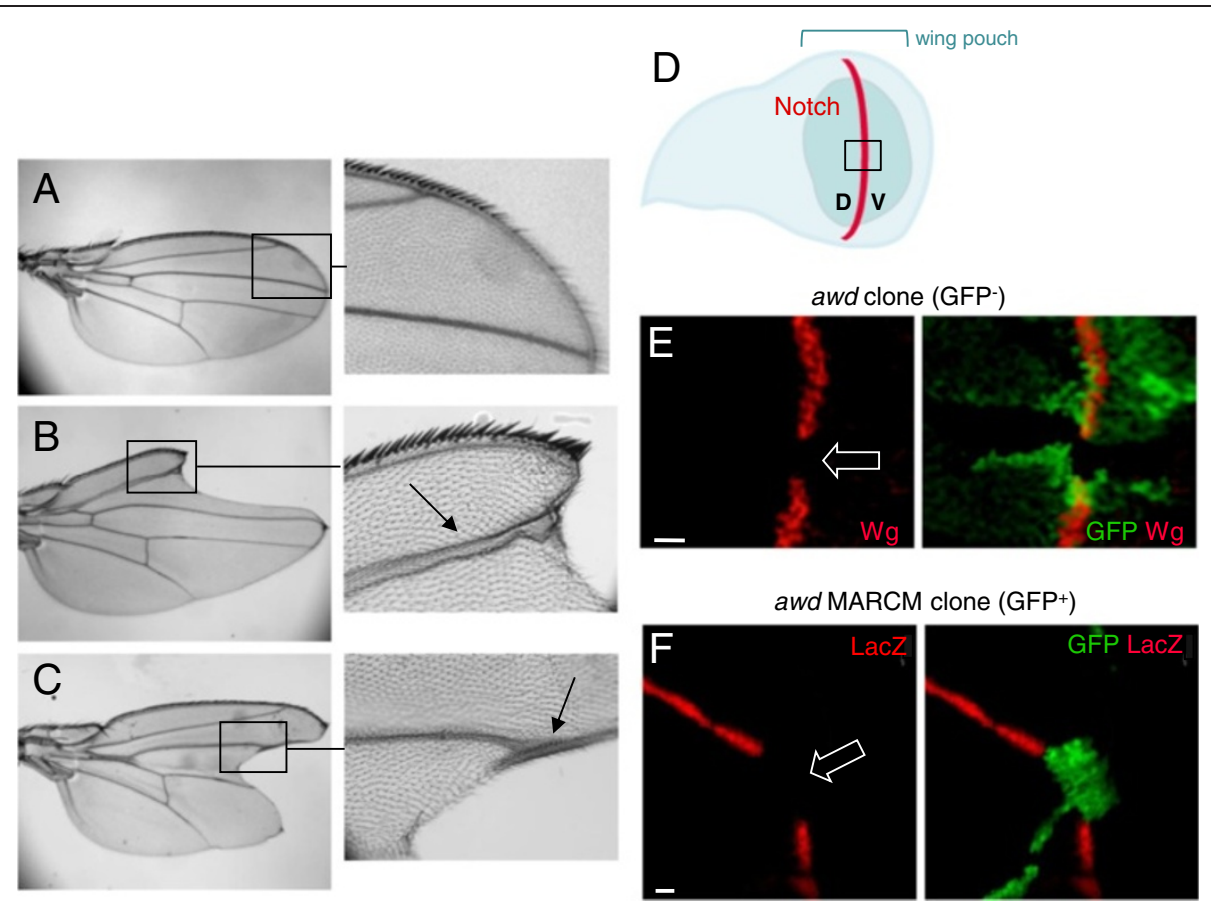

Figure 3 Notch signaling defect in adult wings and larval wing discs. Compared to yw flies, representing wild-type (A), wings from flies of the genotype en2.4-Gal4 $4^{222 C}, U A S-f l p /+; F R T^{82 B} / F R T^{82 B}, a w d^{224}$ (B-C) show typical Notch phenotypes: enlarged wing veins (arrows) and loss of wing margins ('notched' wing blades). (D) Drawing of a third instar wing disc in apical view showing the dorsal-ventral ( $D, V$ ) compartment border (red line) specified by the Notch activity. The wing disc pouch is the central fold of the disc (green) and will generate the wing blade. The black box approximately indicates the areas shown in $\mathrm{E}$ and $\mathrm{F}$. (E) The discs were dissected from $h s-f l p ;+/ 4 ; F R T^{82 B}$, Ubi-GFP/FRT $T^{82 B}$, awd ${ }^{i 2 A 4}$ third instar larvae. wingless (wg) is a downstream activation target of notch. Wg protein expression is lost in the awd clone (loss of GFP; empty block arrow) overlapping the midline (dorsal-ventral boundary, where Notch specifies wg expression). (F) The discs were dissected from hs-flp/GbeSu(H) ms-lacZ; act-Gal4, UAS-GFP/+; $F R T^{22 B}$, act-Gal80/FRT ${ }^{22 B}$, awd ${ }^{2 A 4}$ third instar larvae. GbeSu(H) ms -lacZ expression (red) is also lost in awd MARCM clones (expressing GFP; empty block arrow). Bars are $10 \mu \mathrm{m}$.

mediated transcytosis [61] as well as to internalize Notch for signaling [55]. Since awd mutant cells do not show these very large aggregates throughout the cells, it is unlikely that awd function completely overlaps with that of dynamin. Notch localization can also be influenced by the integrity of the adherens junction [61]. Since we have shown previously that the awd mutant can affect the membrane localization of E-cadherin and $\beta$-catenin [25], we also determined that Notch localization defect not only occurred in awd mutant pile-up epithelial cells [see Additional file 3: Figure S3] but also occurred in awd mutant follicle cells that show normal epithelial polarity, indicated by normal E-cadherin localization (Figure 5E). awd mutant clones exhibiting normal epithelial integrity are most often observed in clones of small size $(<10$ cells; unpublished observation). We showed that small awd mutant clones indeed lacked Hnt expression [see Additional file 4: Figure S4]. We also showed that the epithelial polarity of $a w d$ mutant cells in wing disc is unaffected as shown by normal E-cadherin localization (Figure 5F,G in which $\mathrm{GFP}^{+}$cells are awd mutants). Since Notch processing in the follicle cells has been shown to occur during transition from mature early endosomes to late endosomes [55,62], we suspected that the endocytosis defect in awd mutant cells might be in the step prior to the formation of late endosomes.

To verify this notion, we first examined Notch localization in the endocytic pathway in awd mutant cells. In $a w d^{+}$cells, NICD is in small punctates with partial co-localization with Avl, a component of the early endosome (Figure 6A, upper panels), consistent with previous observations [34,55]. In awd mutant cells, the level of Notch-Avl colocalization increased by 2 fold (Figure 6A, bottom panels; statistical analysis reported in Additional file 5: Figure S5A, $\mathrm{A}^{\prime}$ ).

In order to determine whether these Avl-positive, Notch-containing vesicles are immature early endosomes that cannot form multivesicular bodies (MVBs), we examined the awd mutant vesicles in relation to hepatocyte growth factor-regulated tyrosine kinase substrate (Hrs), which is involved in the maturation of early endosomes by promoting ubiquitinated cargo sorting [63]. It marks the mature early endosomes and MVBs. We observed similar, low-level co-localization of Notch and Hrs in both $a w d^{+}$and awd mutant cells [see Additional file 5: Figure S5B,B' for statistical 


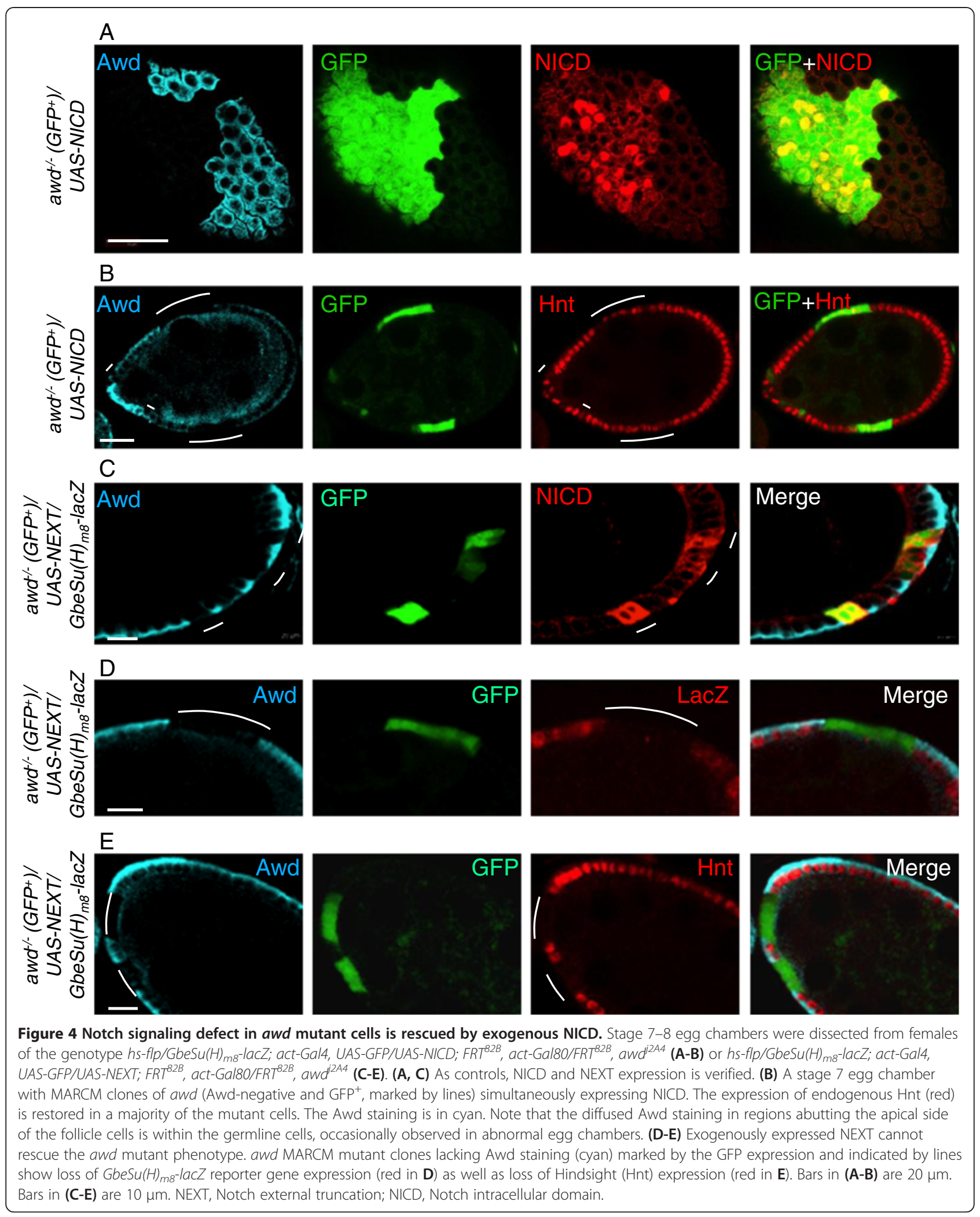




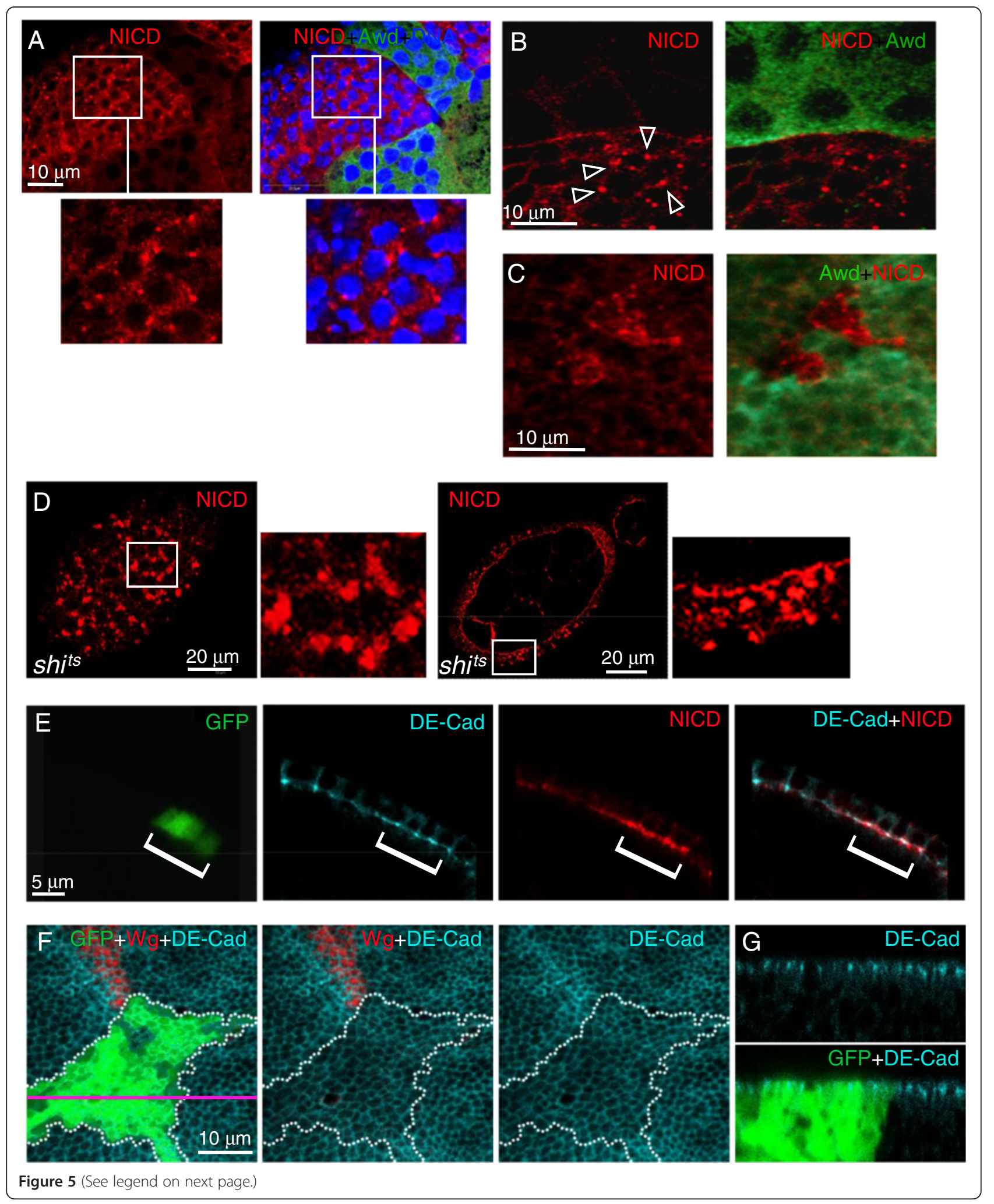


(See figure on previous page.)

Figure 5 Defective intracellular distribution of Notch in awd mutant cells. (A-B) Stage 8 egg chambers were dissected from hs-flp; $+/+$; Ubi-GFP, FRT ${ }^{82 B} / F R T^{82 B}$, awd $d^{i 2 A 4}$ females, and stained for NICD (red), Awd (green) and DNA (blue). Notch over-accumulates in vesicles near the cell periphery (insets in (A) and arrowheads in (B)). (C) The wing disc was dissected from hs-flp; $+/+; \quad U b i-G F P, F R T^{82 B} / F R T^{82 B}, a w d^{j 2 A 4}$ third instar larva and stained for NICD (red). awd clones were identified by lack of Awd staining (pseudo-colored in green). Notch in awd mutant clones accumulates in large vesicles. (D) Surface and cross-section views of shits stage 7 egg chambers from females incubated at $29^{\circ} \mathrm{C}$ and stained for $\mathrm{NICD}$. Very large aggregates are seen on the surface and throughout the cells. (E) A stage 7 egg chamber from a hs-flp/GbeSu(H) $m 8^{-l a c Z ; ~ a c t-G a l 4, ~}$ UAS-GFP/+; FRT ${ }^{82 B}$, act-Gal80/FRT ${ }^{82 B}$, awd ${ }^{22 A 4}$ female was stained for DE-cadherin (cyan) and NICD (red). Notch accumulates in awd mutant cells (GFP-positive) that show normal DE-cadherin distribution. (F-G) Third instar wing imaginal disc dissected from a hs-flp/+; act-Gal4, UAS-GFP/+; $F R T^{82 B}$, act-Gal80/FRT ${ }^{82 B}$, awd ${ }^{2 A 4}$ larva and stained for DE-cadherin (cyan) and Wg (red) in which the awd mutant clone is marked by GFP expression and outlined in $\mathrm{F}$ by the dotted area. (F) The confocal section of the apical region of disc cells ( $\mathrm{x}$-y) shows that awd loss of function does not affect the distribution of DE-cadherin. (G) The cross section through the disc epithelium ( $\mathrm{x}$ - $\mathrm{z}$ ) with apical side up also shows an unaffected apical/basal polarity distribution of DE-cadherin in awd mutant cells. The pink line indicates the position of the $x-z$ section. Awd, Abnormal wing discs; NICD, Notch intracellular domain.

analysis]. Lack of significant Notch-Hrs co-localization even in $a w d^{+}$cells is consistent with the finding that normal Notch signaling is not affected in hrs mutants [55]. Some co-localization of Hrs and Notch in awd mutant cells is also consistent with the observation that a minor Rab5-independent route exists for Notch sorting [55]. On the other hand, this Notch accumulation pattern is very different from that of the phyllopod mutation which blocks Notch entry into late endosomes but not entry into mature early endosomes, resulting in increased Notch signaling and significant co-localization of NICD and Hrs [64]. This suggests that early endosome maturation is defective in awd mutant cells.

Since awd can also act on the internalization of surface receptor [21], we examined whether constitutive internalization of full-length Notch is affected in awd mutant cells. This was detected by using an antibody against the NECD. As shown in Figure 6B, NECD antibody indeed detected increased accumulation of fulllength Notch in awd mutant cells. Therefore, Awd can act on both internalization of surface Notch and intracellular trafficking of signaling Notch.

\section{Notch does not traffic to late endosomes in awd mutant cells}

It has been shown that Notch signaling can also be enhanced by blocking MVB formation with mutations in the endosomal sorting complex required for transport (ESCRT) genes tsg101, vps 25 and vps20, or by promoting early endosome maturation with over-expression of constitutively active Rab5 [55]. Since the awd mutant is defective in Notch signaling, it is unlikely that the Notch-containing vesicles in awd mutant cells have passed into late endosomes. This notion is supported by the lack of significant co-localization of Notch-containing vesicles in MARCM awd mutant clones with Rab7, the lateendosomal marker (GFP-positive cells in Figure 6C; statistical analysis reported in Additional file 6: Figure S6). As well, transition from early endosomes to late endosomes is accompanied by acidification of the luminal contents, which can be detected by Lysotracker staining. Consistent with the notion that Notch-containing vesicles in awd mutant cells cannot enter MVB and late endosomes, we observed no difference in Lysotracker-positive vesicles in $a w d^{+}$ and awd mutant cells [see Additional file 7: Figure S7]. In addition, the Notch-containing vesicles in MARCM awd mutant clones are not Rab11-positive recycling endosomes, either (GFP-positive cells in Figure 6D; see Additional file 8: Figure S8 for statistical analysis).

We next sought to follow the time course of Notch localization in live cells. Wing discs are an ideal and standardized system for this purpose since they can be cultured ex vivo for a prolonged period of time. Note that in this established ex vivo system, internalization of Notch is detected by binding to NECD antibody, without binding to spatially-expressed ligands. Therefore, the system strictly measures the kinetics of vesicular transport, not the endogenous signaling process. We first established that at the steady state (time 0), Notch accumulated on the $a w d^{-/-}$cell surface (Figure 7A). In wild-type cells, internalized Notch follows a typical time course: at 20 minutes after initiation of endocytosis, Notch is mostly in Avl-positive early endosomes while some has passed into Rab7-positive late endosomes (Figure 7B). At one hour after endocytosis, the Notch signal is barely detectable, consistent with the degradation time course. Also, in wild-type cells, Avl staining is much more pronounced at 20 minutes than at one hour. This is likely because in this label-and-chase experiment, a large number of Avl-positive vesicles were formed synchronously after initiation of endocytosis. Concentrated Avl was then lost (therefore detected at a lesser extent by immunofluorescence) after early endosomes matured and were incorporated into late endosomes.

In awd mutant, on the other hand, accumulated Notch is mostly on cell surface or in Avl-positive early endosomes at 20 minutes and remains in these early endosomes even one hour after internalization (Figure 7C). The Notch signal shows no localization to the late 

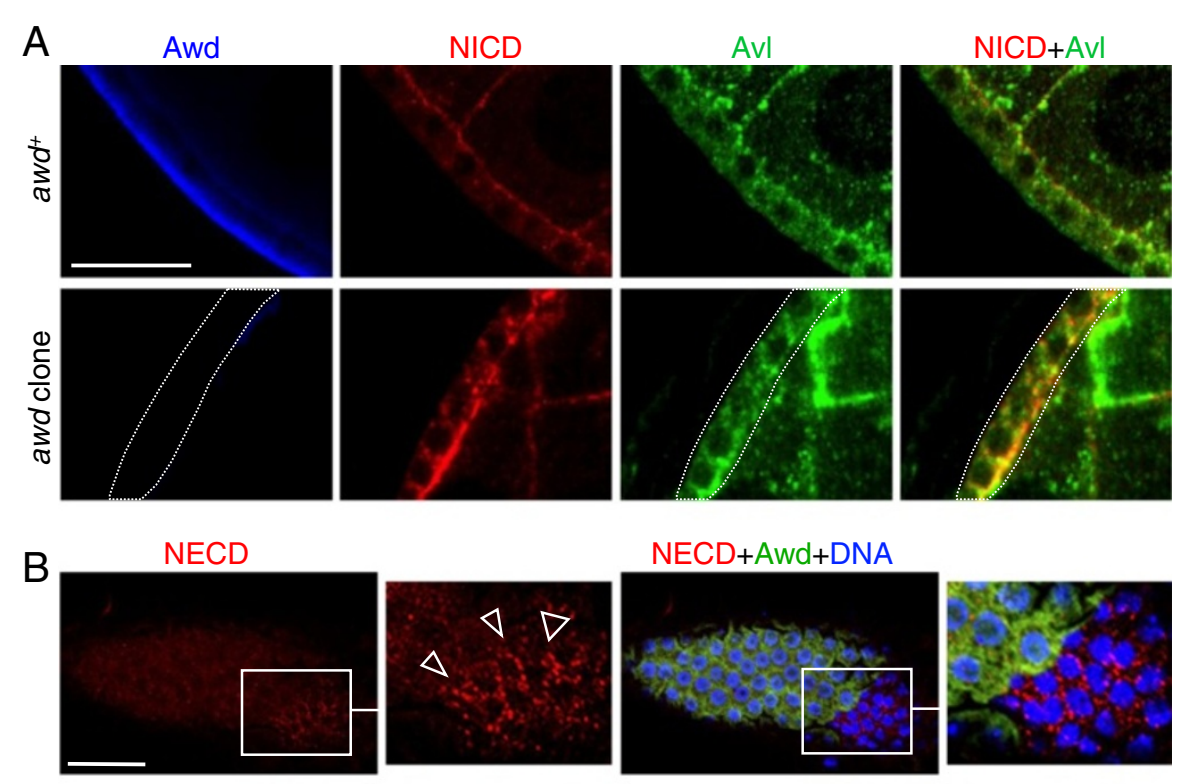

NECD+Awd+DNA
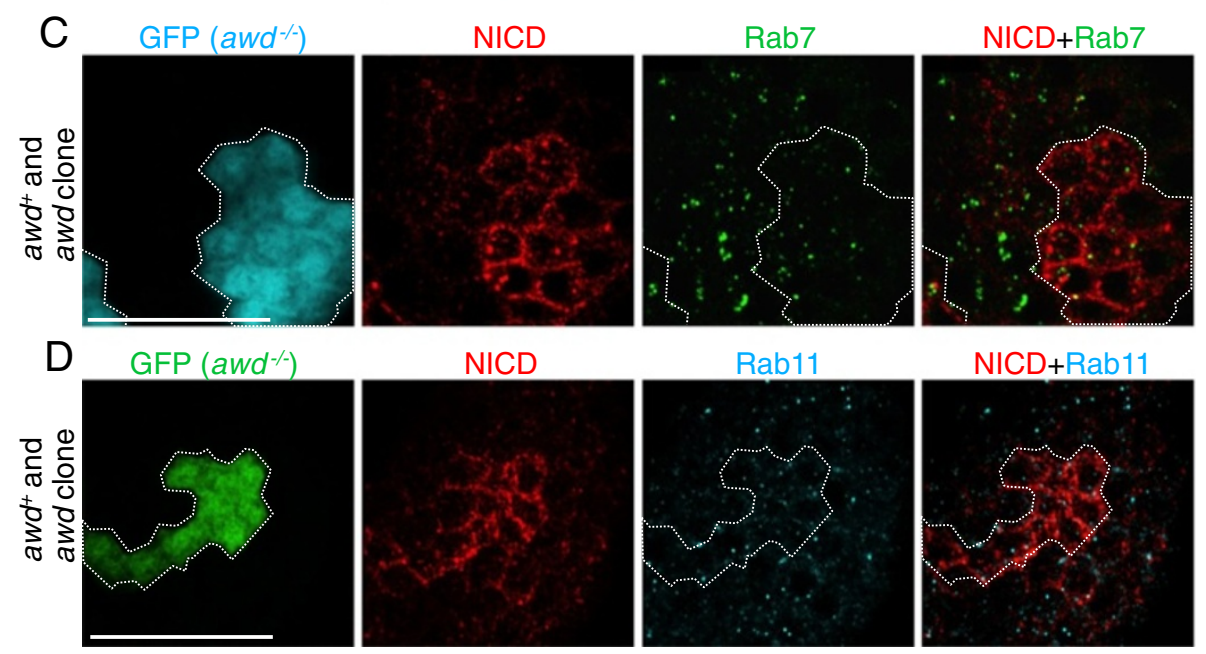

Figure 6 Notch accumulates in early endosomal compartments in awd mutant cells. (A) Stage 8 egg chambers were dissected from yw

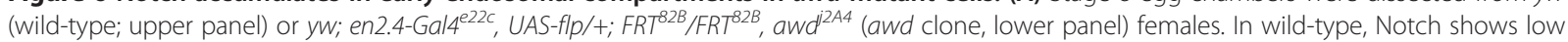
level punctates that are partially co-localized with Avl. In the awd clone, most, if not all, large Notch-positive vesicles are also Avl-positive. Dashed line marks the awd clone. (B) Surface view of stage 7 egg chamber dissected from a yw; en2.4-Gal4 ${ }^{e 22 C}, U A S-f l p /+; F R T^{82 B} / F R T^{82 B}$, awd ${ }^{22 A 4}$ female and stained for Notch extracellular domain peptide (NECD), Awd and DNA. There is accumulation of NECD on the surface of awd mutant clones (empty arrowheads). (C-D) Stage 8 egg chambers were dissected from hs-flp/GbeSu(H) ms - lacZ; act-Gal4, UAS-GFP/t; FRT ${ }^{82 B}$, act-Gal80/FRT ${ }^{82 B}$, awd ${ }^{2 A 4}$ females. awd mutant clones were identified as GFP-expressing cells. In awd clones, over-accumulated Notch does not co-localize with Rab7 (C) or Rab11 (D). Bars are 20 Hm. Avl, avalanche; Awd, abnormal wing disc.

endosomes (Figure 7C). Note that some of the Rab7positive vesicles shown in Figure $7 \mathrm{C}$ are very close to or surrounded by the Notch signal but are not overlapping (Figure 7C insets).

\section{awd is required for Rab5 function}

To further test the role of $a w d$ in early endosome maturation, we next tested how expression of constitutively active Rab5 (Rab5 ${ }^{\mathrm{CA}}$ ) might affect Notch localization in awd mutant. As mentioned above, $\mathrm{Rab} 5^{\mathrm{CA}}$ has been shown to increase Notch signaling [55], presumably because the endocytic process is pushed through early endosomes. In $a w d^{+}$cells, NICD is found in both Rab5 ${ }^{\mathrm{CA}}$-positive (Figure 8A, insets 2 and 3) and -negative (Figure 8A, insets 1 and 4) vesicles, and, importantly, the detectable NICD is almost exclusively in the lumen of these vesicles. The likely interpretation is that $\mathrm{Rab} 5{ }^{\mathrm{CA}}$ pushes endocytosis through early endosomal stages and Notch is processed. Processed endogenous NICD becomes diffused in the cytosol and nuclei, and undetectable by immunohistochemistry (IHC) in our assay system. Remaining, predominantly luminal, NICD is an unprocessed subpopulation that is internalized 


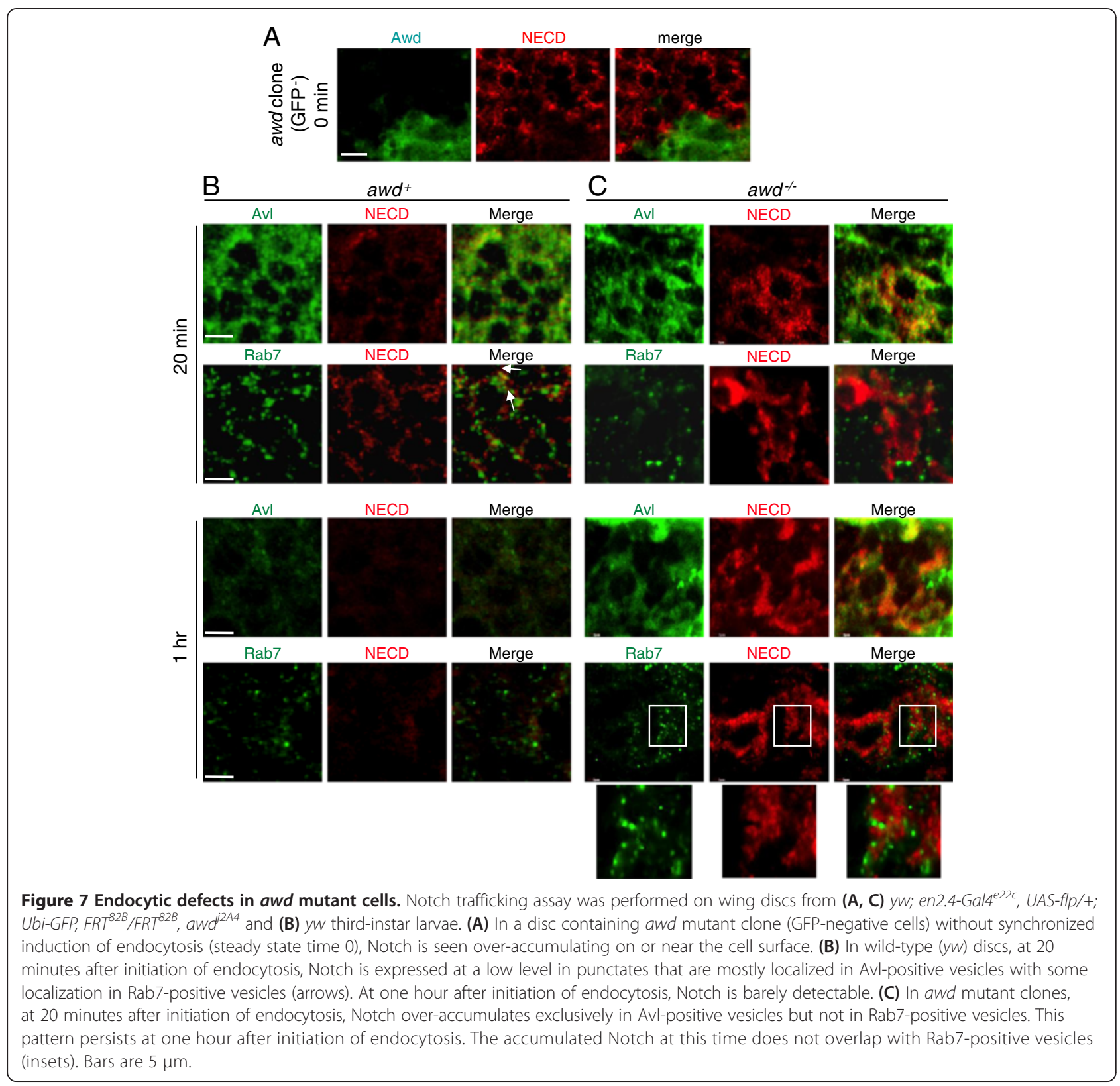

in the MVBs or late endosomes destined for degradation $[31,62]$. Strikingly, in awd mutant clones, NICD is found exclusively in the Rab5 ${ }^{\mathrm{CA}}$-positive vesicles (Figure $8 \mathrm{~B}$ ). Most importantly, in awd mutant cells, much of the NICD signal is mostly present on the surface of these enlarged vesicles (Figure 8B insets $1-3$ ). The result indicates that although cell surface-bound Notch can be internalized in awd mutant cells in the presence of Rab5 $5^{\mathrm{CA}}$, it is not processed and cannot enter late endosomes. In addition, in awd mutant follicle cells $87.1 \%$ of Notch vesicles co-localize with $\operatorname{Rab5} 5^{\mathrm{CA}}$ and $31.45 \%$ co-localize with Hrs $(\mathrm{n}=124)$ (Figure 8C; see Additional file 9: Figure S9 for colocalization analysis). Co-localization of NICD and Hrs in awd mutant cells increases by the over-expression of
$\operatorname{Rab5}{ }^{\mathrm{CA}}$ [see Additional file 9: Figure S9B compared to Additional file 5: Figure S5B']. This suggests that Rab5 ${ }^{\mathrm{CA}}$ partially stimulates vesicles to progress through the endocytic pathway but awd function is necessary for Rab5mediated early endosome maturation. This notion is supported by the increased number of $\mathrm{Rab} 5^{\mathrm{CA}}$-positive vesicles in awd mutant clones (Figure 8B), indicating a block in vesicle trafficking downstream of Rab5 function. This interpretation is confirmed since Rab5 ${ }^{\mathrm{CA}}$ cannot rescue Notch signaling in awd mutant cells (Figure 8D).

Taken together, these results suggest that during Notch signaling awd function is downstream of or is required for Rab5 function in promoting maturation of early endosomes. 

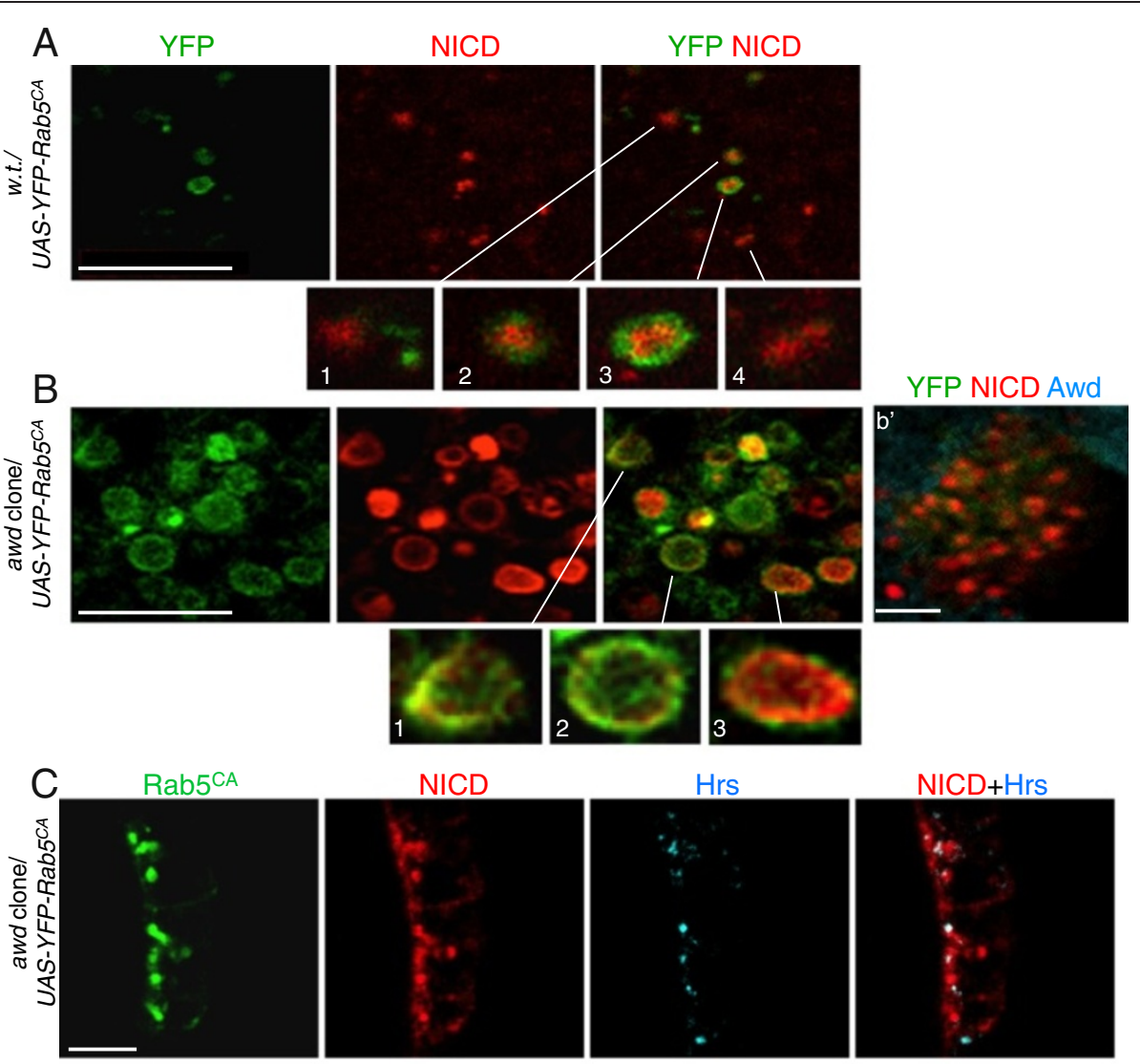

$\mathrm{NICD}+\mathrm{HrS}$
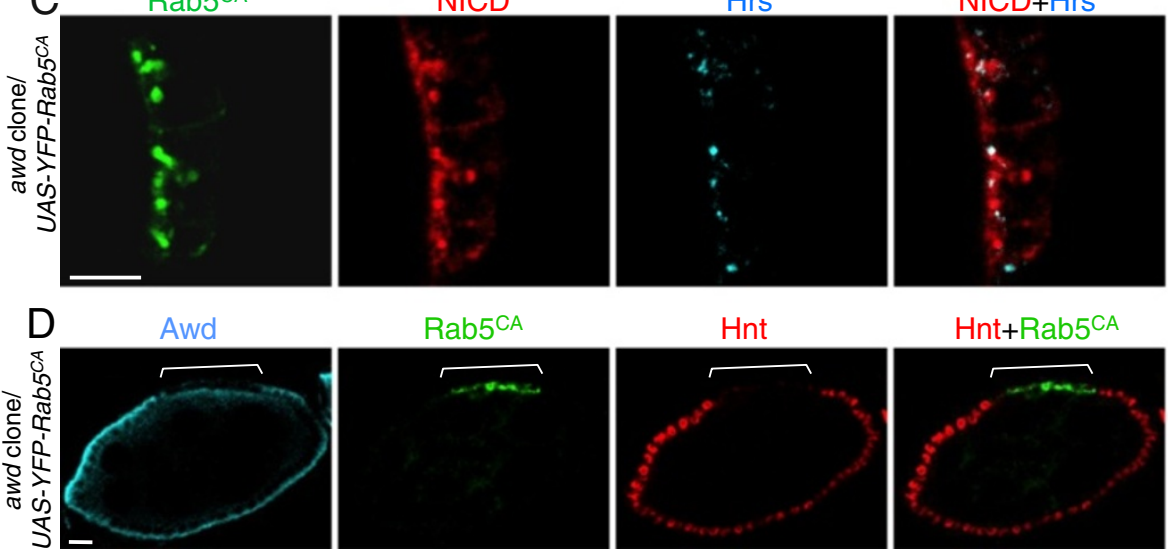

Hnt+Rab5 $5^{\text {A }}$

Figure 8 Awd is required for Rab5 function. YFP-tagged constitutively active Rab5 mutant Q88L (Rab5 $5^{\mathrm{CA}}$ ) were expressed in (A) wild-type or

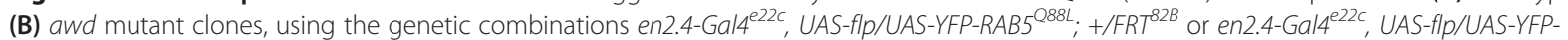
$R A B 5^{Q 88 L}$; awd ${ }^{12 A 4}, F R T^{82 B} / F R T^{82 B}$, respectively. Egg chambers were processed for staining for NICD (red) and YFP (green) as indicated. awd mutants were verified by lack of Awd staining (cyan in $\mathbf{b}^{\prime}$ ). (A) YFP-Rab5 ${ }^{C A}$ expressed in wild-type follicle cells. In Rab5 ${ }^{\text {CA }}$-expressing wild-type follicle cells, NICD is reduced and is present in either Rab5-positive (insets 2 and 3) or Rab5-negative (likely late endosomes; insets 1 and 4). Note that NICD is in the lumen of these vesicles. (B) YFP-Rab5 ${ }^{\mathrm{CA}}$ expressed in awd mutant follicle cells. In Rab5 ${ }^{\mathrm{CA}}$-expressing awd mutant cells, abundant NICD is present in enlarged vesicles that are mostly Rab5-positive. NICD is enriched on the surface of these vesicles (insets 1-3). (C) A stage 8 egg chamber from hs-flp/GbeSu(H) ms-lacZ; UAS-YFP-Rab5 $5^{\mathrm{CA}} /+$; tub-Gal4, FRT ${ }^{82 B}$, tub-Gal80/FRT ${ }^{82 B}$, awd ${ }^{2 A 4}$ was stained for Hrs (cyan), YFP (green) and NICD (red). There is only partial co-localization of accumulated Notch with Hrs. (D) A stage 8 egg chamber from hs-flp/GbeSu(H) mb $^{-l a c Z ;}$ UAS-YFP-Rab5 $5^{C A} /+$; tub-Gal4, FRT $T^{82 B}$, tub-Gal80/FRT $T^{82 B}$, awd ${ }^{j 2 A 4}$ was stained for Awd (cyan), YFP (green) and Hnt (red). Expression of Rab5 ${ }^{\mathrm{CA}}$ in Awd-negative cells (bracket) cannot rescue the loss of Hnt expression. Note that the Awd positive signal apical to the awd mutant clone is the expression sometimes detectable in the germ cell abutting the awd mutant clones. Bars are $10 \mu \mathrm{m}$. Hrs, hepatocyte growth factor-regulated tyrosine kinase substrate; NICD, Notch intracellular domain; YFP, yellow fluorescent protein.

\section{Discussion}

In this report we demonstrate a role of $a w d$ in regulating Notch signaling via its endocytic function including surface internalization and vesicle trafficking. This conclusion is based on our results that show: (1) multiple Notch target genes are mis-expressed in follicle cells and wing discs; (2) Notch accumulates in enlarged early endosomes; and (3) awd function is required for the
Rab5 activity in early endosome maturation. Our results also indicate that during vesicles trafficking, the Awd action is downstream of the S2 cleavage, since overexpressed of NEXT accumulated intracellularly and could not rescue the awd defect. The same NEXT over-expression strategy could rescue the shi/dynamin defect $[57,65]$, strongly supporting the notion that the Awd action on Notch signaling is post-membrane 
invagination. Since over-expression of NICD could rescue the awd defect, the Awd action is likely upstream or in parallel to the S3 cleavage event $(\gamma$-secretase activity). Although a role of awd in promoting the activity of $\gamma$-secretase cannot be completely ruled out, we considered this possibility unlikely. First, awd is a known endocytic factor demonstrated in multiple tissues including neurons, trachea, and follicle cells [22-25]. Second, neither the expression level nor the expression pattern of Presenilin, the catalytic subunit of $\gamma$-secretase, is altered in awd mutant cells. Third, if the defect is in $\gamma$-secretase function, it would be expected that Notch should accumulate in Hrs-positive MVBs [60]. On the contrary, we did not observe such ectopic accumulation of Notch in Hrs-positive vesicles. Therefore, our results, in aggregate, suggest that the main action of Awd on Notch signaling is via its endocytic activity promoting the transition from early endosomes to late endosomes. However, potential defects downstream of $\gamma$-secretase cleavage, such as trafficking to nucleus, in awd mutant cannot be formally ruled out.

One curious exception for the awd function in relation to Notch signaling is found in the border cells. As we have recently reported [24], during the migration of these cells, Awd expression is down-regulated. Re-expression of Awd can lead to reduction of surface receptors, such as PVR that is critical for directional movement, resulting in defective migration. Interestingly, Notch signaling is also important for border cell migration [66]. It, therefore, appears that Notch signaling in these specialized cells does not require Awd activity or is insensitive to Awd protein levels. To test this, we compared Notch expression in border cells with or without Awd re-expression. In wild-type border cells (no Awd), Notch is located on the cell surface as well as in the cell body, consistent with active signaling (data not shown). Forced re-expression of Awd in the border cells does not alter this pattern. This may be because Notch is already actively internalized; increasing the Awd level cannot further enhance such activity. Indeed, endocytosis is intrinsically highly active in border cells $[24,67]$. Alternatively, the differential dependence of Notch on Awd activity may be a function of how Notch is activated, not how Awd functions differently in different cell types. For example, Dobens et al. [68] have shown that the Notch ligand Delta may be co-expressed with Notch in the same border cells. Recent reports have hinted that the requirement of endocytosis for Notch signaling may depend on the ligand-receptor relationship (for example, ligand-dependent or -independent, trans- or cis-activation, and so on) [62]. We, therefore, consider that the apparent Awd-independent Notch signaling in border cells has more to do with the intrinsic Notch signaling mechanism in these cells, and less to do with the function of Awd.
Our results indicate that the Notch signaling defect in awd mutant cells is the failure to deliver Notch past the Rab5-dependent early endosomal stage. On the other hand, the ESCRT complex mutants, which are defective in late endosome formation, promote Notch signaling [34,55]. Taken together, it appears that Notch activation occurs in the intermediate stage between early endosome formation and late endosome entry. Transition from early endosomes to late endosomes is accompanied by cargo sorting, intravesicular invagination and acidification of the luminal contents. Curiously, the matured early endosome and MVB marker hrs mutant has no effect on Notch signaling [55], which indicates that endosomal cargo sorting per se is not required for Notch signaling. We have also shown that awd mutant cells do not exhibit altered levels of Lysotracker staining and that endosomal Notch remains on the surface of enlarged endosomes in awd mutants. The exact nature of this transition state that favors Notch processing, therefore, requires further analysis. The endocytic function of awd has traditionally been described as a 'GTP supplier' for Dynamin, based on genetic interaction data and logical extrapolation because of the GTP producing activity of Awd [22]. In this report, we demonstrate that, in relation to Notch signaling, awd functions downstream of, but not directly on, dynamin. It is instead critical for Rab5 activity. This is supported by the following evidence: 1) Notch in awd mutant accumulates in Avl-containing vesicles. Therefore, the awd defect is post Dynamin-mediated cleavage of membrane invagination. 2) $R a b 5^{C A}$ can push Notch into enlarged early endosomes but failed to rescue the awd phenotype, thereby strengthening the notion that awd defect is post Shi/Dynamin function. 3) The Notch accumulation pattern in shi mutant is different from that in awd mutant. 4) Over-expression of NEXT could not rescue awd defect. The same NEXT over-expression strategy could rescue the shi defect, strongly supporting the notion that the Awd action concerning Notch signaling is post-membrane invagination $[57,65]$. It should be noted that we did observe surface accumulation of NECD antibody-detected Notch molecules, likely representing the full-length Notch not engaged in ligand binding and signaling. This indicates that Awd can affect constitutive internalization of full-length Notch.

The requirement of endocytosis in the signal-receiving cells for Notch activation has been amply demonstrated [69]. It has been shown that Notch signaling in follicle cells after stage 6 requires Delta [42]. Since in this report we show that Notch signaling cannot occur in the follicle cell without awd function, we conclude that, at least in follicle cells, endocytosis is a requisite process for ligand-dependent Notch signaling.

The involvement of endocytosis in Notch signaling is significant since many of the endocytic components shown to regulate Notch signaling have also been implicated in 
carcinogenesis. For example, V-ATPase is required for Notch signaling while mutations in ESCRT components, such as Tsg101, result in increased Notch signaling. VATPase has generally been considered an oncogene [70] because it is associated with acidification of tumor cells. ESCRT components, on the other hand, have been shown to suppress tumor formation because they downregulate surface growth factor receptor signaling [71]. As such, attempts to design therapeutics based on these prevalent functions should take into account the effects on Notch signaling, since the relationship between Notch signaling and carcinogenesis is context-dependent [35,72,73].

\section{Conclusions}

Awd belongs to the Nm23 family of protein that is evolutionarily conserved from Drosophila to mammals. Our in vivo analyses demonstrate that loss of awd gene function blocks Notch signaling by altering the receptor processing after the S2 cleavage and causes Notch accumulation in early endosomes. Furthermore, we obtained evidence indicating that Awd is required for Rab5 function in early endosome formation.

$N m 23$ has been an enigmatic gene function. It is a housekeeping gene involved in nucleotide synthesis and energy metabolism, and yet exhibiting specific developmental functions [6,24]. It was the first metastasis suppressor gene identified [4,74], yet exhibits oncogenic functions in some cancer cohorts $[9,10]$. We have previously shown that either loss-of-function or over-expression of awd can affect different aspects of epithelial morphogenesis. That is, lossof-function awd results in over-accumulation of adherens junction components and piling up of the epithelium, while over-expression of awd results in reduced adherens junctions and disintegration of epithelial structure [25]. These findings provided some explanation of the biphasic function of Nm23 in tumorigenesis. In light of the studies presented here, an additional level of complexity should be considered since Notch signaling can exert different cellular functions in different tissues and at different times during pathophysiological alterations of the same tissues [35].

\section{Methods}

\section{Drosophila strains and genetics}

Stocks were raised on standard cornmeal/yeast/agar medium at $25^{\circ} \mathrm{C}$. The stock carrying the protein-null awd allele, $a w d^{j 2 A 4}$, has been described [22-25]. The $a w d^{j 2 A 4}$ allele combined with the FRT chromosome $F R T^{82 B}$ has been described [24,25]. Cell clones mutant for $a w d^{2 A 4}$ were generated through mitotic recombination using the FLP/FRT system [44], either with the hs-flp recombinase transgene or using the directed mosaic technique with the UAS-flp transgene under control of the ubiquitous somatic cell driver en2.4-Gal4 ${ }^{22 c}$ [45]. To obtain over-expression of specific transgenes in $a w d^{2 A 4}$ mutant follicle cells we used either the directed mosaics or the MARCM [46] techniques. The transgenic line carrying the constitutively active (CA) variant of the YFP-Rab5 fusion genes was obtained from the Bloomington Stock Center (Bloomington, IN, USA) [75]. The UAS-NICD and the $G b e S u(H)_{m 8}-l a c Z$ lines were a kind gift from S. Bray of University of Cambridge (Cambridge, UK). The UAS-NEXT line was a kind gift from M. Fortini of Thomas Jefferson University (Philadelphia, PA, USA). The genotypes of flies and larvae used for the analyses are described in Additional file 10; Supplementary experimental procedures.

\section{Immunohistochemistry}

Ovaries were dissected, fixed and stained as previously described [76] with the exception of ovaries from $s h i^{2} / s h i^{2}$ $\left(s h i^{t s}\right)$ females that were fixed at $29^{\circ} \mathrm{C}$. Whole late third instar larvae were dissected into room temperature PBS $(\mathrm{pH}$ 7.5), and fixed for 20 minutes in $4 \%$ formaldehyde. After three washes in PBS, larval tissues were permeabilized for one hour in PBT (0.3\% Triton X-100 in PBS) and then were blocked in $2 \% \mathrm{BSA}$ in $\mathrm{PBT}$ for 10 minutes at room temperature. Overnight incubation at $4^{\circ} \mathrm{C}$ with primary antibodies in 2\% BSA in PBT was followed by three washes in PBT and 10 minutes incubation in 2\% BSA in PBT. Larval samples were then incubated with fluorescence-tagged secondary antibodies for two hours at room temperature and after extensive washes in PBT the wing discs were dissected. Primary antibodies used are: chicken anti-Avl (1:500) [59], mouse monoclonal anti-NICD (1:1000; C17.9c6, Developmental Studies Hybridoma Bank (DSHB, Iowa City, Iowa, USA)), mouse monoclonal anti-NECD (1:50; C458.2H, DSHB), mouse monoclonal anti-Cut (1:15; 2B10, DSHB), mouse monoclonal anti-Hnt (1:30; 1G9, DSHB), mouse monoclonal anti-Cyclin B (1:100; F2F4, DSHB), rat monoclonal anti-DE-cadherin (1:100; DCAD2, DSHB) and mouse monoclonal anti- $\beta$-gal $(1: 25 ; 40-1 \mathrm{~A}$, DSHB); and protein A-purified rabbit anti-Awd (1:2000) [23], rabbit anti-phosphohistone H3 (1:200; 06-570, Upstate Biotechnology, Lake Placid, NY, USA), rabbit anti-C-Psn (1:200) [58], rabbit anti-Rab7 (1:2000) and rabbit antiRab11 (1:8000) [77]. Secondary antibodies used are: Cy3(1:100; Jackson Lab, West Grove, PA, USA), DyLight 649- (1:200; Jackson Lab), or FITC- (1:250; Invitrogen, Molecular Probes, Eugene, OR, USA) conjugated antimouse immunoglobulin G (IgG); and Cy3- (1:1000; Sigma, Saint Louis, Missouri, USA), DyLight 649- (1:500; Jackson Lab), or BODIPY- (1:2000; Molecular Probes) conjugated anti-rabbit IgG.

DNA staining was carried out by incubating egg chambers and wing discs for 10 minutes with 4,6-diamidino2-phenylindole (DAPI; Sigma) at $0.5 \mu \mathrm{g} / \mathrm{ml}$ in PBS followed by several washes with PBS. To-Pro-3 (Molecular Probes) nuclear staining was also carried out after immunodetection by incubating the egg chambers for 
two hours with To-Pro-3 at $1 \mu \mathrm{M}$ in PBS on a rotating wheel followed by several washes with PBT. Stained egg chambers or wing discs were mounted in Fluoromount-G (Electron Microscopy Sciences, Hatfield, PA, USA) and were subsequently analyzed with conventional epifluorescence on a Nikon Eclipse 90i microscope and with a TCS SL Leica confocal system. Digital images were processed and assembled using the Adobe Photoshop software. No biased image manipulations were applied.

\section{Cuticle preparation of adult wings}

Adult flies of the genotype en2.4-Gal4 ${ }^{e 22 c}, U A S-f l p /+$; $F R T^{82 B} / F R T^{82 B}$, awd $d^{j 2 A 4}$ were collected. Wings were removed at the hinge, dehydrated in ethanol and mounted on microscope slides in lactic acid/ethanol (6:5). Wing images were captured by a Nikon Eclipse 90i microscope and acquired with a Nikon Digital Sight camera.

\section{Notch endocytosis assay}

The assay was adopted from a published report [78] with modifications. Third instar larval wing discs were dissected in Schneider's Drosophila medium (SDM) containing 1\% fetal calf serum. The discs were cultured for 15 minutes on ice in the presence of the mouse monoclonal anti-NECD antibody. Excess antibody was rinsed away and the discs were incubated with fresh media at room temperature. The discs were dissected at different times and detected with anti mouse IgG.

\section{Co-localization and statistical analysis}

Thresholds of confocal images were set in Adobe Photoshop to exclude background staining. Images were processed with the CDA plugin of ImageJ to obtain the Pearson's coefficient. Statistical comparison was performed by two-tailed paired Student's t-test (GraphPad Prism 6 software).

\section{Lysotracker staining}

For Lysotracker ex vivo staining, females were dissected in SDM. Ovaries were collected, separated and incubated in medium containing $5 \mu \mathrm{M}$ Lysotracker (DND-99, Molecular Probes) in soft agitation for five minutes at room temperature in the dark. Ovaries were then rapidly washed three times with fresh SDM, mounted and imaged immediately.

\section{Additional files}

Additional file 1: Figure S1. Notch signaling in wild type follicle cells is upregulated by either NICD or NEXT over-expression. Females of the genotype hs-flp, UAS-mCD8GFP/act > CD2 > Gal4; +/UAS-NICD (A) or hs-flp, UAS-mCD8GFP/act > CD2 > Gal4; + /UAS-NEXT (B) were dissected and the egg chambers were stained for Hnt (red). Over-expression of NICD at stage 7-8 in wild type follicle cells marked by GFP expression (green) enhances the level of Hnt expression in $51 \%$ of follicle cells $(n=100)$. Over-expression of NEXT at stage 7-8 in wild type follicle cells marked by the expression of GFP (green) enhances the level of Hnt expression in 92.5\% of follicle cells $(n=40)$. Bars are $15 \mu \mathrm{m}$.

Additional file 2: Figure S2. Presenilin expression pattern is not altered in awd mutant follicle cells. Polyclonal rabbit antibody against a Cterminal peptide in the putative hydrophilic loop region of Psn (anti-CPsn) has been described [58]. Stage 6 and 7 egg chambers containing MARCM clones of awd mutant (marked by positive GFP expression) were stained for Psn (cyan) and NICD (red). Psn is ubiquitously expressed in intracellular punctates in both follicle cells and germ cells. No changes in either the expression level or the punctate pattern are observed in awd mutant cells. The egg chambers were dissected from hs-flp/GbeSu(H) ms-lacZ; act-Gal4, UAS-GFP/+; FRT ${ }^{82 B}$, act-Gal80/FRT ${ }^{82 B}$, awd ${ }^{12 A 4}$ females. Bars are $5 \mu \mathrm{m}$.

Additional file 3: Figure S3. Disrupted epithelial cells in awd mutant clone show abnormal Notch accumulation. Females of the genotype en2.4-Gal4 ${ }^{222 C}, U A S-f l p /+; F R T^{82 B} / F R T^{82 B}$, awd $d^{2 A 4}$ were dissected and the egg chambers were stained for DNA (DAPI), Awd, NICD, and Avl as indicated. Abnormal Notch accumulation in large vesicles is observed in pile-up mutant epithelial cells (arrows), which co-localize with the early endosomal marker Avl (see also Additional file 5: Figure S5A, $\mathrm{A}^{\prime}$ ). Bar is $20 \mu \mathrm{m}$.

Additional file 4: Figure S4. Small awd mutant clones exhibit loss of Hnt expression. Stage 7-8 egg chambers were dissected from hs-flp/ GbeSu(H) ms-lacZ; act-Gal4, UAS-GFP/t; FRT $T^{82 B}$, act-Gal80/FRT $T^{82 B}$, awd $d^{2 A 4}$ females and stained for Hnt (red) and DNA (cyan). Quantitative analysis of Hnt expression was perfomed in awd clones (GFP-positive cells, green) containing a maximum of 5 cells. In these small clones $93 \%$ of awd mutant cells lack Hnt expression $(n=42)$. Bar are $5 \mu \mathrm{m}$.

Additional file 5: Figure S5. Analysis of Notch vesicle co-localization with Avl and Hrs. In awd mutant cells, Notch accumulates in Avl-positive and Hrs-negative early endosomes. Stage 7-8 egg chambers were dissected from hs-flp/GbeSu(H) m8-lacZ; act-Gal4, UAS-GFP/t; FRT ${ }^{82 B}$, act-Gal80/ $F R T^{82 B}, a w d^{i 2 A 4}$ females and stained for NICD and Avl $\left(\mathbf{A}, \mathbf{A}^{\prime}\right)$ or NICD and Hrs (B, $\left.\mathbf{B}^{\prime}\right)$. Co-localization was analyzed by using ImageJ. The Pearson's coefficient ranges from $+1=$ complete correlation to $-1=$ anti-correlation, with $0=$ no correlation. The mean values $(n=4)$ of Pearson's coefficients for NICD and Avl (A) and for NICD and Hrs (B) in $a w d^{+}$and awd mutant cells were plotted together with standard deviations (error bars). Statistical significance was calculated using the two-tailed paired t-test ${ }^{* *}=P$ $<0.01 ;$ N.S. = No Significant). (A') Co-localization image of NICD and Avl based on the analysis of awd mutant cells and neighboring awd cells showed in Figure 6A. (B') Co-localization image of NICD and Hrs based on the analysis of awd mutant cells and neighboring $a w d^{+}$cells.

Additional file 6: Figure S6. Analysis of Notch vesicle co-localization with Rab7. In awd mutant cells, Notch does not accumulate in Rab7positive endosomes. Stage 7-8 egg chambers were dissected from hs-flp/ GbeSu(H) ms-lacZ; act-Gal4, UAS-GFP/t; FRT $T^{82 B}$, act-Gal80/FRT $T^{82 B}, a_{w d} d^{2 A 4}$ females and stained for NICD and Rab7. Co-localization was analyzed by using ImageJ. The mean values $(n=6)$ of Pearson's coefficients for NICD and Rab7 in awd $d^{+}$and awd mutant cells were plotted together with standard deviations (error bars) (A). Statistical significance was calculated using the two-tailed paired t-test (N.S. = Not Significant). (A') Co-localization image of NICD and Rab7 based on the analysis of awd mutant cells and neighboring awd ${ }^{+}$cells showed in Figure 6C.

Additional file 7: Figure S7. $a w d^{+}$and awd mutant cells show similar Lysotracker staining patterns. The egg chambers were dissected from hs-flp/GbeSu(H) ms-lacZ; act-Gal4, UAS-GFP/t;FRT ${ }^{82 B}$, act-Gal80/FRT ${ }^{82 B}$, awd $d^{2 A 4}$ females and stained for Lysotracker. GFP expression identifies awd mutant clones. There is no difference in acidified endosomal compartments between awd and awd mutant cells. Bar is $5 \mu \mathrm{m}$.

Additional file 8: Figure S8. Analysis of Notch vesicle co-localization with Rab11. In awd mutant cells, Notch does not accumulate in Rab11positive endosomes. Stage 7-8 egg chambers were dissected from hs-flp/ GbeSu(H) ms-lacZ; act-Gal4, UAS-GFP/+; FRT ${ }^{82 B}$, act-Gal80/FRT ${ }^{82 B}$, awd $d^{2 A 4} \mathrm{fe}-$ males and stained for NICD and Rab11. Co-localization was analyzed by using ImageJ. The mean values $(n=4)$ of Pearson's coefficients for NICD and Rab11 in awd $^{+}$and awd mutant cells were plotted together with standard deviations (error bars) (A). Statistical significance was calculated using the two-tailed paired t-test (N.S. = Not Significant). (A') Co- 
localization image of NICD and Rab11 based on the analysis of awd mutant cells and neighboring awd ${ }^{+}$cells showed in Figure 6D.

Additional file 9: Figure S9. Analysis of Notch vesicle co-localization with Rab5 ${ }^{\mathrm{CA}}$ and Hrs. Stage 7-8 egg chambers were dissected from hsflp/GbeSu(H) ms-lacZ; UAS-YFP-Rab5 1 / + t tub-Gal4, FRT ${ }^{82 B}$, tub-Gal80/FRT ${ }^{82 B}$ awd $d^{2 A 4}$ females and stained for NICD and Hrs. Quantitative analysis of Notch vesicle co-localization with Rab5 ${ }^{\mathrm{CA}}$ and Hrs was performed. In awd mutant cells $87.1 \%$ of Notch vesicles co-localizes with Rab5 ${ }^{\text {CA }}$ and $31.45 \%$ co-localizes with $\mathrm{Hrs}(\mathrm{n}=124)$. Co-localization was analyzed also by using ImageJ. The mean values $(n=6)$ of Pearson's coefficients for NICD and $\operatorname{Rab5}^{\mathrm{CA}}(\mathrm{A})$ and for NICD and Hrs (B) in awd mutant cells are reported with standard deviations. (A) Co-localization image of NICD and Rab5 ${ }^{\text {CA }}$ and (B) co-localization image of NICD and Hrs are based on the analysis of awd mutant cells showed in Figure $8 \mathrm{C}$.

\section{Additional file 10: Supplementary experimental procedures.}

\section{Abbreviations}

Avl: Avalanche; Awd: abnormal wing discs; BSA: bovine serum albumin CSL: CBF1-Su(H)-Lag1; DSL: Delta/Serrate/Lag2; ESCRT: endosomal sorting complex required for transport; Faslll: Fasciclin III; FGFR: fibroblast growth factor receptor; GbeSu(H) ms: Grainyhead transcription factor binding site, Suppressor of Hairless binding sites, Enhancer of split m8 gene; GFP: green fluorescent protein; Hnt: Hindsight; Hrs: hepatocyte growth factor-regulated tyrosine kinase substrate; lgG: immunoglobulin G; MARCM: mosaic analysis with a repressible cell marker; MVBs: multivesicular bodies; NDPK: nucleoside diphosphate kinase; NECD: Notch extracellular domain; NEXT: Notch External Truncation; NICD: Notch intracellular domain; Nm23/NME: non metastatic cells; NRR: negative regulatory region; PBS: phosphate-buffered saline; PBT: Triton-100 in PBS; PDGF: platelet-derived growth factor; PVR: PDGF/NEGF receptor; Rab5 ${ }^{\mathrm{CA}}$ : constitutively active Rab5; SDM: Schneider's Drosophila medium; Shi: Shibire; UAS: upstream activating sequence; VEGF: vascular endothelial growth factor; YFP: yellow fluorescent protein.

\section{Competing interests}

The authors declare that they have no competing interests.

\section{Authors' contributions}

$\mathrm{MI}, \mathrm{MB}, \mathrm{GN}$, JW and SD performed the experiments and participated in the discussion and conception part of the experiments. VC, GG and TH participated in the discussion, conceived and designed the experiments. VC and TH wrote the manuscript. All authors read and approved the final manuscript.

\section{Acknowledgements}

This paper is dedicated to the memory of our wonderful colleague Dr. Franco Graziani, a devoted scientist and great motivating mentor. We thank S. Bray, M. Fortini, M. Blanco, T. Vaccari, D. Bilder, A. Nakamura and B. Lemaitre for generous gifts of fly stocks and antibodies. We also thank the Bloomington Stock Center for providing us with fly stocks and the Developmental Studies Hybridoma Bank at the University of lowa for monoclonal antibodies. We are particularly grateful to Carlo Taddei for his encouragements and insightful discussions throughout the course of this work. We thank P. Romani for helpful suggestions on preparing the manuscript. We also thank Marco Privitera for his graphic work. The work was supported by a research grant from the Association for International Cancer Research (AICR grant ref. 11-0738) to V.C., a grant from University of Bologna (RFO 2009) to G.G. and V.C., and a grant from the National Institutes of Health (R01CA109860) to T.H.

\section{Author details}

${ }^{1}$ Dipartimento di Farmacia e Biotecnologie, Alma Mater Studiorum Università di Bologna, Via Selmi, 3, Bologna 40126, Italy. ${ }^{2}$ Department of Medicine, Boston University School of Medicine, Boston, Massachusetts 02118, USA. ${ }^{3}$ Department of Pathology and Laboratory Medicine, Medical University of South Carolina, Charleston, South Carolina 29425, USA. ${ }^{4}$ Graduate Institute of Systems Biology and Bioinformatics, National Central University, Jhongli, Taiwan. ${ }^{5}$ Present address: Institute of Hematology "L. e A. Seràgnoli", University of Bologna, Bologna, Italy. ${ }^{6}$ Present address: Bone Regeneration Laboratory, Research Institute Codivilla-Putti, Rizzoli Orthopaedic Institute, Bologna, Italy.
Received: 5 August 2013 Accepted: 10 February 2014

Published: 14 February 2014

\section{References}

1. Dearolf CR, Hersperger E, Shearn A: Developmental consequences of awdb3, a cell-autonomous lethal mutation of Drosophila induced by hybrid dysgenesis. Dev Biol 1988, 129:159-168.

2. Dearolf CR, Tripoulas N, Biggs J, Shearn A: Molecular consequences of awdb3, a cell-autonomous lethal mutation of Drosophila induced by hybrid dysgenesis. Dev Biol 1988, 129:169-178.

3. Rosengard AM, Krutzsch HC, Shearn A, Biggs JR, Barker E, Margulies IM, King CR, Liotta LA, Steeg PS: Reduced Nm23/Awd protein in tumour metastasis and aberrant Drosophila development. Nature 1989, 342:177-180.

4. Steeg PS, Bevilacqua G, Kopper L, Thorgeirsson UP, Talmadge JE, Liotta LA, Sobel ME: Evidence for a novel gene associated with low tumor metastatic potential. J Natl Cancer Inst 1988, 80:200-204.

5. Desvignes T, Pontarotti P, Fauvel C, Bobe J: Nme protein family evolutionary history, a vertebrate perspective. BMC Evol Biol 2009, 9:256.

6. Hsu T: NME genes in epithelial morphogenesis. Naunyn Schmiedebergs Arch Pharmacol 2011, 384:363-372.

7. Onyenwoke RU, Forsberg LJ, Liu L, Williams T, Alzate O, Brenman JE: AMPK directly inhibits NDPK through a phosphoserine switch to maintain cellular homeostasis. Mol Biol Cell 2011, 23:381-389.

8. Ouatas T, Salerno M, Palmieri D, Steeg PS: Basic and translational advances in cancer metastasis: Nm23. J Bioenerg Biomembr 2003, 35:73-79.

9. Harlozinska A, Bar JK, Gerber J: nm23 expression in tissue sections and tumor effusion cells of ovarian neoplasms. Int J Cancer 1996, 69:415-419.

10. Mandai M, Konishi I, Koshiyama M, Mori T, Arao S, Tashiro H, Okamura H, Nomura $\mathrm{H}$, Hiai H, Fukumoto M: Expression of metastasis-related nm23-H1 and $\mathrm{nm} 23-\mathrm{H} 2$ genes in ovarian carcinomas: correlation with clinicopathology, EGFR, c-erbB-2, and c-erbB-3 genes, and sex steroid receptor expression. Cancer Res 1825-1830, 1994:54.

11. Steeg PS, Zollo M, Wieland T: A critical evaluation of biochemical activities reported for the nucleoside diphosphate kinase/Nm23/Awd family proteins: opportunities and missteps in understanding their biological functions. Naunyn Schmiedebergs Arch Pharmacol 2011, 384:331-339.

12. Wagner PD, Steeg PS, Vu ND: Two-component kinase-like activity of $\mathrm{nm} 23$ correlates with its motility-suppressing activity. Proc Natl Acad Sci U S A 1997, 94:9000-9005.

13. Inoue H, Takahashi M, Oomori A, Sekiguchi M, Yoshioka T: A novel function for nucleoside diphosphate kinase in Drosophila. Biochem Biophys Res Commun 1996, 218:887-892.

14. Engel M, Veron M, Theisinger B, Lacombe ML, Seib T, Dooley S, Welter C: A novel serine/threonine-specific protein phosphotransferase activity of Nm23/nucleoside-diphosphate kinase. Eur J Biochem 1995, 234:200-207.

15. Ma D, Xing Z, Liu B, Pedigo NG, Zimmer SG, Bai Z, Postel EH, Kaetzel DM: $\mathrm{NM} 23-\mathrm{H} 1$ and $\mathrm{NM} 23-\mathrm{H} 2$ repress transcriptional activities of nucleasehypersensitive elements in the platelet-derived growth factor- $\mathrm{A}$ promoter. J Biol Chem 2002, 277:1560-1567.

16. Postel EH, Berberich SJ, Rooney JW, Kaetzel DM: Human NM23/nucleoside diphosphate kinase regulates gene expression through DNA binding to nuclease-hypersensitive transcriptional elements. J Bioenerg Biomembr 2000, 32:277-284.

17. Fan Z, Beresford PJ, Oh DY, Zhang D, Lieberman J: Tumor suppressor NM23-H1 is a granzyme A-activated DNase during CTL-mediated apoptosis, and the nucleosome assembly protein SET is its inhibitor. Cell 2003, 112:659-672.

18. Zhang Q, McCorkle JR, Novak M, Yang M, Kaetzel DM: Metastasis suppressor function of NM23-H1 requires its 3'-5' exonuclease activity. Int J Cancer 2011, 128:40-50.

19. Tokarska-Schlattner M, Boissan M, Munier A, Borot C, Mailleau C, Speer O, Schlattner U, Lacombe ML: The nucleoside diphosphate kinase D (NM23-H4) binds the inner mitochondrial membrane with high affinity to cardiolipin and couples nucleotide transfer with respiration. J Biol Chem 2008, 283:26198-26207.

20. Epand RF, Schlattner U, Wallimann T, Lacombe ML, Epand RM: Novel lipid transfer property of two mitochondrial proteins that bridge the inner and outer membranes. Biophys J 2007, 92:126-137.

21. Nallamothu G, Dammai V, Hsu T: Developmental function of Nm23/awd: a mediator of endocytosis. Mol Cell Biochem 2009, 329:35-44.

22. Krishnan KS, Rikhy R, Rao S, Shivalkar M, Mosko M, Narayanan R, Etter P, Estes PS, Ramaswami M: Nucleoside diphosphate kinase, a source of GTP, 
is required for dynamin-dependent synaptic vesicle recycling. Neuron 2001, 30:197-210.

23. Dammai V, Adryan B, Lavenburg KR, Hsu T: Drosophila awd, the homolog of human $n m 23$, regulates FGF receptor levels and functions synergistically with shi/dynamin during tracheal development. Genes Dev 2003, 17:2812-2824.

24. Nallamothu G, Woolworth JA, Dammai V, Hsu T: Awd, the homolog of metastasis suppressor gene $\mathrm{Nm} 23$, regulates Drosophila epithelial cell invasion. Mol Cell Biol 1964-1973, 2008:28

25. Woolworth JA, Nallamothu G, Hsu T: The Drosophila metastasis suppressor gene $\mathrm{Nm} 23$ homolog, awd, regulates epithelial integrity during oogenesis. Mol Cell Biol 2009, 29:4679-4690.

26. Artavanis-Tsakonas S, Rand MD, Lake RJ: Notch signaling: cell fate control and signal integration in development. Science 1999, 284:770-776.

27. Mumm JS, Schroeter EH, Saxena MT, Griesemer A, Tian X, Pan DJ, Ray WJ, Kopan R: A ligand-induced extracellular cleavage regulates gammasecretase-like proteolytic activation of Notch1. Mol Cell 2000, 5:197-206.

28. Le Borgne R: Regulation of Notch signalling by endocytosis and endosomal sorting. Curr Opin Cell Biol 2006, 18:213-222.

29. Bray SJ: Notch signalling: a simple pathway becomes complex. Nat Rev Mol Cell Biol 2006, 7:678-689.

30. Tien AC, Rajan A, Bellen HJ: A Notch updated. J Cell Biol 2009, 184:621-629.

31. Le Borgne R, Bardin A, Schweisguth F: The roles of receptor and ligand endocytosis in regulating Notch signaling. Development 2005, 132:1751-1762.

32. Moberg KH, Schelble S, Burdick SK, Hariharan IK: Mutations in erupted, the Drosophila ortholog of mammalian tumor susceptibility gene 101, elicit non-cell-autonomous overgrowth. Dev Cell 2005, 9:699-710.

33. Thompson BJ, Mathieu J, Sung HH, Loeser E, Rorth P, Cohen SM: Tumor suppressor properties of the ESCRT-II complex component Vps25 in Drosophila. Dev Cell 2005, 9:711-720.

34. Vaccari T, Bilder D: The Drosophila tumor suppressor vps 25 prevents nonautonomous overproliferation by regulating notch trafficking. Dev Cell 2005, 9:687-698

35. South AP, Cho RJ, Aster JC: The double-edged sword of Notch signaling in cancer. Semin Cell Dev Biol 2012, 23:458-464.

36. Roth S, Lynch JA: Symmetry breaking during Drosophila oogenesis. Cold Spring Harb Perspect Biol 2009, 1:a001891.

37. Wu X, Tanwar PS, Raftery LA: Drosophila follicle cells: morphogenesis in an eggshell. Semin Cell Dev Biol 2008, 19:271-282.

38. Klusza S, Deng WM: At the crossroads of differentiation and proliferation: precise control of cell-cycle changes by multiple signaling pathways in Drosophila follicle cells. Bioessays 2011, 33:124-134.

39. Dobens LL, Raftery LA: Integration of epithelial patterning and morphogenesis in Drosophila ovarian follicle cells. Dev Dyn 2000, 218:80-93.

40. Sun J, Deng WM: Notch-dependent downregulation of the homeodomain gene cut is required for the mitotic cycle/endocycle switch and cell differentiation in Drosophila follicle cells. Development 2005, 132:4299-4308.

41. Deng WM, Althauser C, Ruohola-Baker H: Notch-Delta signaling induces a transition from mitotic cell cycle to endocycle in Drosophila follicle cells. Development 2001, 128:4737-4746.

42. Lopez-Schier H, St Johnston D: Delta signaling from the germ line controls the proliferation and differentiation of the somatic follicle cells during Drosophila oogenesis. Genes Dev 2001, 15:1393-1405.

43. Shcherbata HR, Althauser C, Findley SD, Ruohola-Baker H: The mitotic-toendocycle switch in Drosophila follicle cells is executed by Notchdependent regulation of $\mathrm{G} 1 / \mathrm{S}, \mathrm{G} 2 / \mathrm{M}$ and M/G1 cell-cycle transitions. Development 2004, 131:3169-3181.

44. Xu T, Rubin GM: Analysis of genetic mosaics in developing and adult Drosophila tissues. Development 1993, 117:1223-1237.

45. Duffy JB, Harrison DA, Perrimon N: Identifying loci required for follicular patterning using directed mosaics. Development 1998, 125:2263-2271.

46. Lee T, Luo L: Mosaic analysis with a repressible cell marker for studies of gene function in neuronal morphogenesis. Neuron 1999, 22:451-461.

47. Sun J, Deng WM: Hindsight mediates the role of notch in suppressing hedgehog signaling and cell proliferation. Dev Cell 2007, 12:431-442.

48. Furriols M, Bray S: A model Notch response element detects Suppressor of Hairless-dependent molecular switch. Curr Biol 2001, 11:60-64.

49. de Celis JF, Garcia-Bellido A, Bray SJ: Activation and function of Notch at the dorsal-ventral boundary of the wing imaginal disc. Development 1996 122:359-369.
50. Sanchez-Irizarry C, Carpenter AC, Weng AP, Pear WS, Aster JC, Blacklow SC: Notch subunit heterodimerization and prevention of ligand-independent proteolytic activation depend, respectively, on a novel domain and the LNR repeats. Mol Cell Biol 2004, 24:9265-9273.

51. Schroeter EH, Kisslinger JA, Kopan R: Notch-1 signalling requires ligand-induced proteolytic release of intracellular domain. Nature 1998, 393:382-386.

52. Rebay I, Fehon RG, Artavanis-Tsakonas S: Specific truncations of Drosophila Notch define dominant activated and dominant negative forms of the receptor. Cell 1993, 74:319-329.

53. Go MJ, Eastman DS, Artavanis-Tsakonas S: Cell proliferation control by Notch signaling in Drosophila development. Development 2031-2040, 1998:125.

54. Sun J, Smith L, Armento A, Deng WM: Regulation of the endocycle/gene amplification switch by Notch and ecdysone signaling. J Cell Biol 2008, 182:885-896

55. Vaccari T, Lu H, Kanwar R, Fortini ME, Bilder D: Endosomal entry regulates Notch receptor activation in Drosophila melanogaster. J Cell Biol 2008 180:755-762.

56. Ye Y, Fortini ME: Characterization of Drosophila Presenilin and its colocalization with Notch during development. Mech Dev 1998, 79:199-211.

57. Lopez-Schier H, St Johnston D: Drosophila nicastrin is essential for the intramembranous cleavage of notch. Dev Cell 2002, 2:79-89.

58. Nowotny P, Gorski SM, Han SW, Philips K, Ray WJ, Nowotny V, Jones CJ, Clark RF, Cagan RL, Goate AM: Posttranslational modification and plasma membrane localization of the Drosophila melanogaster presenilin. Mol Cell Neurosci 2000, 15:88-98.

59. Lu H, Bilder D: Endocytic control of epithelial polarity and proliferation in Drosophila. Nat Cell Biol 2005, 7:1232-1239.

60. Yan Y, Denef N, Schupbach T: The vacuolar proton pump, V-ATPase, is required for notch signaling and endosomal trafficking in Drosophila. Dev Cell 2009, 17:387-402.

61. Sasaki N, Sasamura T, Ishikawa HO, Kanai M, Ueda R, Saigo K, Matsuno K: Polarized exocytosis and transcytosis of Notch during its apical localization in Drosophila epithelial cells. Genes Cells 2007, 12:89-103.

62. Wilkin $M$, Tongngok $\mathrm{P}$, Gensch $\mathrm{N}$, Clemence $\mathrm{S}$, Motoki M, Yamada K, Hori K, Taniguchi-Kanai M, Franklin E, Matsuno K, Baron M: Drosophila HOPS and AP-3 complex genes are required for a Deltex-regulated activation of notch in the endosomal trafficking pathway. Dev Cell 2008, 15:762-772.

63. Komada M, Kitamura N: The Hrs/STAM complex in the downregulation of receptor tyrosine kinases. J Biochem 2005, 137:1-8.

64. Nagaraj R, Banerjee U: Regulation of Notch and Wingless signalling by phyllopod, a transcriptional target of the EGFR pathway. EMBO J 2009, 28:337-346.

65. Struhl G, Adachi A: Requirements for presenilin-dependent cleavage of notch and other transmembrane proteins. Mol Cell 2000, 6:625-636.

66. Wang X, Adam JC, Montell D: Spatially localized Kuzbanian required for specific activation of Notch during border cell migration. Dev Biol 2007, 301:532-540.

67. Silver DL, Geisbrecht ER, Montell DJ: Requirement for JAK/STAT signaling throughout border cell migration in Drosophila. Development 2005, 132:3483-3492.

68. Dobens $L$, Jaeger A, Peterson JS, Raftery LA: Bunched sets a boundary for Notch signaling to pattern anterior eggshell structures during Drosophila oogenesis. Dev Biol 2005, 287:425-437.

69. Baron M: Endocytic routes to Notch activation. Semin Cell Dev Biol 2012, 23:437-442

70. Sennoune SR, Martinez-Zaguilan R: Vacuolar H(+)-ATPase signaling pathway in cancer. Curr Protein Pept Sci 2012, 13:152-163.

71. Wegner CS, Rodahl LM, Stenmark H: ESCRT proteins and cell signalling. Traffic 2011, 12:1291-1297.

72. Court H, Amoyel M, Hackman M, Lee KE, Xu R, Miller G, Bar-Sagi D, Bach EA Bergo MO, Philips MR: Isoprenylcysteine carboxylmethyltransferase deficiency exacerbates KRAS-driven pancreatic neoplasia via Notch suppression. J Clin Invest 2013, 123:4681-4681.

73. Guijarro MV, Dahiya S, Danielson LS, Segura MF, Vales-Lara FM, Menendez S, Popiolek D, Mittal K, Wei JJ, Zavadil J, Cordon-Cardo C, Pandolfi PP, Hernando E: Dual Pten/Tp53 suppression promotes sarcoma progression by activating Notch signaling. Am J Pathol 2015-2027, 2013:182.

74. Heimann R, Hellman S: Individual characterisation of the metastatic capacity of human breast carcinoma. Eur J Cancer 2000, 36:1631-1639. 
75. Zhang J, Schulze KL, Hiesinger PR, Suyama K, Wang S, Fish M, Acar M, Hoskins RA, Bellen HJ, Scott MP: Thirty-one flavors of Drosophila rab proteins. Genetics 2007, 176:1307-1322.

76. Duchi S, Fagnocchi L, Cavaliere V, Hsouna A, Gargiulo G, Hsu T: Drosophila VHL tumor-suppressor gene regulates epithelial morphogenesis by promoting microtubule and aPKC stability. Development 2010, 137:1493-1503.

77. Tanaka T, Nakamura A: The endocytic pathway acts downstream of Oskar in Drosophila germ plasm assembly. Development 2008, 135:1107-1117.

78. Le Borgne R, Schweisguth F: Unequal segregation of Neuralized biases Notch activation during asymmetric cell division. Dev Cell 2003, 5:139-148.

doi:10.1186/1741-7007-12-12

Cite this article as: Ignesti et al:: Notch signaling during development requires the function of $a w d$, the Drosophila homolog of human metastasis suppressor gene Nm23. BMC Biology 2014 12:12.

\section{Submit your next manuscript to BioMed Central and take full advantage of:}

- Convenient online submission

- Thorough peer review

- No space constraints or color figure charges

- Immediate publication on acceptance

- Inclusion in PubMed, CAS, Scopus and Google Scholar

- Research which is freely available for redistribution 